\title{
High performance computation for direct numerical and large eddy simulation
}

\author{
Chaoqun Liu*
}

(Received 18 November 2004, revised 25 February 2006)

\begin{abstract}
This paper focusses on high order compact schemes for direct numerical simulation (DNS) and large eddy simulation (LES) for flow separation, transition, tip vortex, and flow control. We discuss the fundamental issues of high quality grid generation, high order schemes for curvilinear coordinates, the CFL condition for complex geometry, and high-order weighted compact schemes for shock capturing and shock-vortex interaction. The computation examples include DNS for K-type and H-type transition, DNS for flow separation and transition around an airfoil with attack angle, control of flow separation by using pulsed jets, and LES simulation for a tip vortex behind the juncture of a wing and flat plate. Computations also show the almost linear growth in efficiency obtained using multiple processors.
\end{abstract}

*Department of Mathematics, University of Texas at Arlington, TX, USA. mailto: cliu@uta.edu

See http://anziamj.austms.org.au/V46/CTAC2004/Liu2 for this article, (C) Austral. Mathematical Soc. 2006. Published March 31, 2006. ISSN 1446-8735 


\section{Contents}

1 Introduction

C1373

2 Numerical approaches for high order DNS in curvilinear coordinates

C1374

2.1 Governing equations . . . . . . . . . . . . . C C1374

2.2 Grids: smoothness, orthogonality, high order Jacobian, conservation for curvilinear coordinates . . . . . . . . . . C1376

2.3 High-order compact schemes . . . . . . . . . . . . . . . C C1379

2.4 Weighted compact scheme . . . . . . . . . . . C C1380

2.4.1 Basic formulations of weighted compact scheme . . C1380

2.4.2 Conservation and reconstruction function . . . . . . C1382

2.5 Linear stability (CFL conditions) for stretched grids . . . . C1382

2.5.1 CFL condition . . . . . . . . . . . . . C1382

2.5.2 CFL condition for stretched and curved grids . . . C1383

2.6 Non-linearity and high-order filter . . . . . . . . . . . C1384

2.6.1 Non-linearity and high-frequency waves . . . . . . . C1384

2.6.2 Low passing filter . . . . . . . . . . . . . . . C1385

2.6.3 Filter and artificial viscosity . . . . . . . . . . C1385

2.7 Fully implicit scheme and iteration (flow solver) . . . . . C1386

2.8 Non reflecting boundary conditions . . . . . . . . . . . C1387

2.9 MPI parallel computation . . . . . . . . . . . . . . . C1389

3 DNS for flow transition

C1390

3.1 Problem definitions and boundary conditions . . . . . . . . C1390

3.2 Computational results . . . . . . . . . . . . . . C C1393

4 DNS for flow separation and control around an airfoil C1396 4.1 Problem definitions and boundary conditions . . . . . . . C1396 4.2 Computational results and analysis . . . . . . . . . C1398 4.2.1 Flow around the airfoil without blowing (baseline case)C1398 4.2.2 Flow around the airfoil with a pulsed blowing jet . C1401 4.2.3 The effect of blowing angle . . . . . . . . . . . C1405 
5 LES for wingtip vortex around juncture of wing and flat plate

C1406

5.1 Grid generation . . . . . . . . . . . . . . . C1406

5.2 Case setup . . . . . . . . . . . . . . . . . . . . C1407

5.3 LES results . . . . . . . . . . . . . . . . . . C1408

6 Conclusions

C1410

References

C1411

\section{Introduction}

Many important practical flow problems are time dependent with a wide range of length scales. Examples include flow transition, separation, turbulence, shock turbulence interaction, wakes, and acoustic waves. These cannot be predicted and understood by traditional Reynolds Averaged NavierStokes (RANS) methods, but are amenable to direct numerical simulation (DNS) or, at least, large eddy simulation (LES). Due to the incredible pace at which computer speed is increasing and computer prices are falling, DNS and LES can nowadays be performed on a cheap PC-cluster which most companies or universities can afford, enabling more and more realistic flow problems to be seriously studied by DNS or LES. Several millions of grids cells with tens of thousands of time steps are now fairly normal for DNS or LES calculations. Meanwhile, advanced computational methodology is urgently sought by researchers to increase the computational efficiency and accuracy. Such methodology includes, in particular, high order schemes, high order filters, high quality grid generation, high order schemes for shock turbulence interaction, fast flow solvers, and parallel computation.

This paper summarizes efforts in utilizing DNS and LES for some of the most challenging flow problems investigated at the Center for Numerical Simulation and Modeling at the University of Texas at Arlington. The re- 
searchers include Dr L. Jiang, Dr H. Shan, Mr S. Deng and Mr J. Cai. We focus on the application of high order schemes for a general geometry.

The paper is arranged as follows: Section 2 mainly discusses numerical approaches for DNS/LES and Sections 3, 4 and 5 provide several DNS/LES computational examples which are challenging in modern fluid dynamics. Section 6 provides some conclusions.

\section{Numerical approaches for high order DNS in curvilinear coordinates}

\section{$2.1 \quad$ Governing equations}

The three dimensional compressible Navier-Stokes equations in generalized curvilinear coordinates $(\xi, \eta, \zeta)$ may be written in conservative form as

$$
\frac{1}{J} \frac{\partial Q}{\partial t}+\frac{\partial\left(E-E_{v}\right)}{\partial \xi}+\frac{\partial\left(F-F_{v}\right)}{\partial \eta}+\frac{\partial\left(G-G_{v}\right)}{\partial \zeta}=0 .
$$

Here the coordinate transformation between the curvilinear $(\xi, \eta, \zeta)$ and Cartesian $(x, y, z)$ frames are defined by

$$
\xi=\xi(x, y, z), \quad \eta=\eta(x, y, z), \quad \zeta=\zeta(x, y, z),
$$

and $J=\frac{\partial(\xi, \eta, \zeta)}{\partial(x, y, z)}$ is the Jacobian of the transformation. The vector of conserved quantities $Q$, the inviscid flux vector $(E, F, G)$, and the viscous flux vector $\left(E_{v}, F_{v}, G_{v}\right)$ are

$$
Q=\left[\begin{array}{c}
\rho \\
\rho u \\
\rho v \\
\rho w \\
E_{t}
\end{array}\right], \quad E=\frac{1}{J}\left[\begin{array}{c}
\rho U \\
\rho U u+p \xi_{x} \\
\rho U v+p \xi_{y} \\
\rho U w+p \xi_{z} \\
U\left(E_{t}+p\right)
\end{array}\right], \quad F=\frac{1}{J}\left[\begin{array}{c}
\rho V \\
\rho V u+p \eta_{x} \\
\rho V v+p \eta_{y} \\
\rho V w+p \eta_{z} \\
V\left(E_{t}+p\right)
\end{array}\right],
$$




$$
\begin{aligned}
& G=\frac{1}{J}\left[\begin{array}{c}
\rho W \\
\rho W u+p \zeta_{x} \\
\rho W v+p \zeta_{y} \\
\rho W w+p \zeta_{z} \\
W\left(E_{t}+p\right)
\end{array}\right], \quad E_{v}=\frac{1}{J}\left[\begin{array}{c}
0 \\
\tau_{x x} \xi_{x}+\tau_{y x} \xi_{y}+\tau_{z x} \xi_{z} \\
\tau_{x y} \xi_{x}+\tau_{y y} \xi_{y}+\tau_{z y} \xi_{z} \\
\tau_{x z} \xi_{x}+\tau_{y z} \xi_{y}+\tau_{z z} \xi_{z} \\
Q_{x} \xi_{x}+Q_{y} \xi_{y}+Q_{z} \xi_{z}
\end{array}\right], \\
& F_{v}=\frac{1}{J}\left[\begin{array}{c}
0 \\
\tau_{x x} \eta_{x}+\tau_{y x} \eta_{y}+\tau_{z x} \eta_{z} \\
\tau_{x y} \eta_{x}+\tau_{y y} \eta_{y}+\tau_{z y} \eta_{z} \\
\tau_{x z} \eta_{x}+\tau_{y z} \eta_{y}+\tau_{z z} \eta_{z} \\
Q_{x} \eta_{x}+Q_{y} \eta_{y}+Q_{z} \eta_{z}
\end{array}\right], \quad G_{v}=\frac{1}{J}\left[\begin{array}{c}
\tau_{x x} \zeta_{x}+\tau_{y x} \zeta_{y}+\tau_{z x} \zeta_{z} \\
\tau_{x y} \zeta_{x}+\tau_{y y} \zeta_{y}+\tau_{z y} \zeta_{z} \\
\tau_{x z} \zeta_{x}+\tau_{y z} \zeta_{y}+\tau_{z z} \zeta_{z} \\
Q_{x} \zeta_{x}+Q_{y} \zeta_{y}+Q_{z} \zeta_{z}
\end{array}\right],
\end{aligned}
$$

where $u, v$ and $w$ are the velocity components in the $x, y$ and $z$ directions respectively, and $\xi_{x}, \xi_{y}, \xi_{z}, \eta_{x}, \eta_{y}, \eta_{z}, \zeta_{x}, \zeta_{y}, \zeta_{z}$ are the coordinate transformation metrics. The contra-variant velocity components are

$$
U=u \xi_{x}+v \xi_{y}+w \xi_{z}, \quad V=u \eta_{x}+v \eta_{y}+w \eta_{z}, \quad W=u \zeta_{x}+v \zeta_{y}+w \zeta_{z}
$$

whereas $Q_{x}, Q_{y}$ and $Q_{z}$ in the energy equation are

$$
\begin{aligned}
& Q_{x}=-q_{x}+u \tau_{x x}+v \tau_{x y}+w \tau_{x z}, \\
& Q_{y}=-q_{y}+u \tau_{x y}+v \tau_{y y}+w \tau_{y z}, \\
& Q_{z}=-q_{z}+u \tau_{x z}+v \tau_{y z}+w \tau_{z z},
\end{aligned}
$$

and $E_{t}$ is the total energy. The components of the viscous stress tensor and heat flux are denoted by $\tau_{x x}, \tau_{y y}, \tau_{z z}, \tau_{x y}, \tau_{x z}, \tau_{y z}$, and $q_{x}, q_{y}, q_{z}$ respectively.

To obtain the dimensionless form, the reference values for length, density, velocity, temperature, pressure and time are $L, \rho_{r}, U_{r}, T_{r}, \rho_{r} U_{r}^{2}$ and $L / U_{r}$ respectively. Here, the free stream parameters are chosen as reference values and the chord length of the airfoil is used as the reference length. The dimensionless parameters that arise are the Mach number $M_{r}$, Reynolds number Re, Prandtl number Pr, and the ratio of the specific heats $\gamma$ :

$$
M_{r}=\frac{U_{r}}{\sqrt{\gamma R T}}, \quad \operatorname{Re}=\frac{\rho_{r} U_{r} L}{\mu_{r}}, \quad \operatorname{Pr}=\frac{C_{p} \mu_{r}}{k_{r}}, \quad \gamma=\frac{C_{p}}{C_{v}},
$$


where $R$ is the ideal gas constant, $C_{p}$ and $C_{v}$ are specific heats at constant pressure and constant volume respectively. Throughout this work, $\operatorname{Pr}=0.7$ and $\gamma=1.4$. Viscosity is determined according to the Sutherland's law, which in dimensionless form is

$$
\mu=\frac{T^{3 / 2}(1+S)}{T+S}, \quad S=\frac{110.3 K}{T_{r}},
$$

where $T$ is the temperature in degrees $\mathrm{K}$.

The governing system is closed by the equation of state:

$$
\gamma M_{r}^{2} p=\rho T, \quad E_{t}=\frac{p}{\gamma-1}+\frac{1}{2} \rho\left(u^{2}+v^{2}+w^{2}\right) .
$$

The components of the viscous stress tensor and heat flux in non-dimensional form are

$$
\tau_{i j}=\frac{\mu}{\operatorname{Re}}\left[\left(\frac{\partial u_{i}}{\partial x_{j}}+\frac{\partial u_{j}}{\partial x_{i}}\right)-\frac{2}{3} \delta_{i j} \frac{\partial u_{k}}{\partial x_{k}}\right], \quad q_{i}=-\frac{\mu}{(\gamma-1) M_{r}^{2} \operatorname{Re} \operatorname{Pr}} \frac{\partial T}{\partial x_{i}} .
$$

\subsection{Grids: smoothness, orthogonality, high order Jacobian, conservation for curvilinear coordinates}

The DNS and LES computation requires a high order accuracy to capture high frequency waves and small magnitude disturbances. It is a challenge to achieve high order accuracy with a body-fitted grid. Let us consider the discretization for the first order derivative of a general function $\varphi(x, y, z)$ :

$$
\begin{aligned}
\frac{\partial \varphi}{\partial x}= & \frac{\partial \varphi}{\partial \xi} \frac{\partial \xi}{\partial x}+\frac{\partial \varphi}{\partial \eta} \frac{\partial \eta}{\partial x}+\frac{\partial \varphi}{\partial \zeta} \frac{\partial \zeta}{\partial x} \\
= & \left(\frac{\delta \varphi}{\delta \xi}+\tilde{\tau}_{\xi}\right)\left(\frac{\delta \xi}{\delta x}+\tilde{\tau}_{1}\right)+\left(\frac{\delta \varphi}{\delta \eta}+\tilde{\tau}_{\eta}\right)\left(\frac{\delta \eta}{\delta x}+\tilde{\tau}_{2}\right) \\
& +\left(\frac{\delta \varphi}{\delta \zeta}+\tilde{\tau}_{\zeta}\right)\left(\frac{\delta \zeta}{\delta x}+\tilde{\tau}_{3}\right) .
\end{aligned}
$$




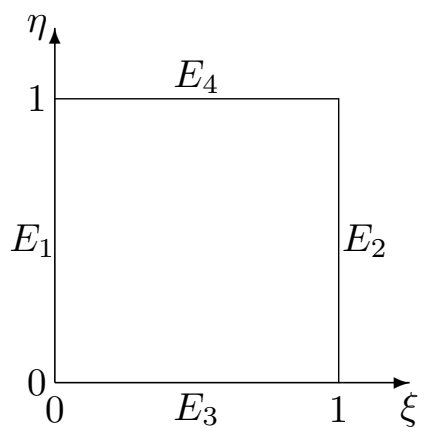

(a)

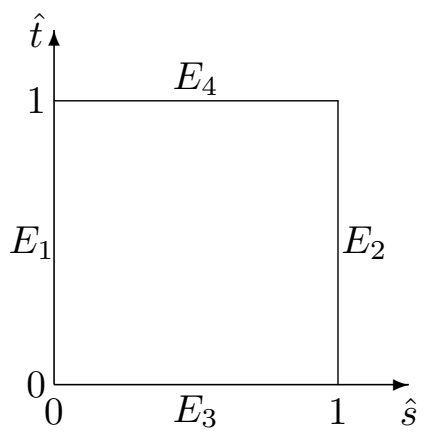

(b)

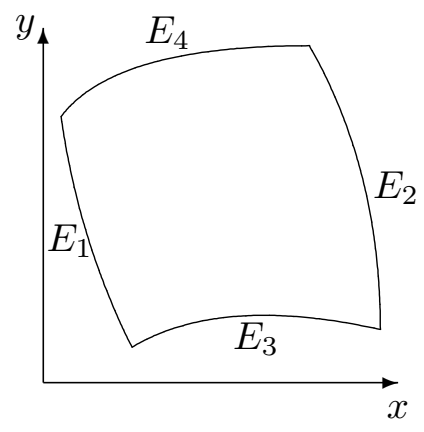

(c)

Figure 1: (a) Computational space $\mathcal{C}$, (b) Parameter space $\mathcal{P}$, and (c) Physical domain $\mathcal{D}$.

Here $\delta \cdot / \delta \xi$ denotes a finite difference approximation of $\partial \cdot / \partial \xi$, and $\tilde{\tau}$ the truncation error. In order to achieve sixth order accuracy of the discretization, we require that the grid functions $\xi, \eta$ and $\zeta$ have at least seventh order derivatives and the discretization of Jacobian has at least sixth order accuracy. For $3 \mathrm{D}$ problems, additional conservation conditions must be satisfied [20]:

$$
\begin{aligned}
& I_{1}=\left(\hat{\xi}_{x}\right)_{\xi}+\left(\hat{\eta}_{x}\right)_{\eta}+\left(\hat{\zeta}_{x}\right)_{\zeta}=0, \\
& I_{2}=\left(\hat{\xi}_{y}\right)_{\xi}+\left(\hat{\eta}_{y}\right)_{\eta}+\left(\hat{\zeta}_{y}\right)_{\zeta}=0, \\
& I_{3}=\left(\hat{\xi}_{z}\right)_{\xi}+\left(\hat{\eta}_{z}\right)_{\eta}+\left(\hat{\zeta}_{z}\right)_{\zeta}=0,
\end{aligned}
$$

where $\hat{\xi}_{x}=J^{-1} \xi_{x}$ and $J=\frac{\partial(\xi, \eta, \zeta)}{\partial(x, y, z)}$.

We must check these conditions after we generate the 3D grids. In order to avoid or reduce the error of discretization of the cross derivatives, an orthogonal grid near the wall surface where the velocity gradient is large is preferred.

An elliptic grid generation method first proposed by Spekreijse [16] generates the grids; it is based on a composite mapping of a nonlinear algebraic transformation and an elliptic transformation. For simplicity, consider 2D 
grid generation to illustrate the method. The algebraic grid transformation maps the computational space $\mathcal{C}$ onto a parameter space $\mathcal{P}$, and the elliptic transformation maps the parameter space on to the physical domain $\mathcal{D}$. Figure 1 illustrates the computational space, parameter space, and the physical domain.

The parameter space $\mathcal{P}$ is defined as a unit space in a two dimensional space with Cartesian coordinate $(\hat{s}, \hat{t})$, and $\hat{s} \in[0,1], \hat{t} \in[0,1]$. The boundary values of $\hat{s}$ and $\hat{t}$ are determined by the grid point distribution in the physical domain:

- $\hat{s}=0$ at edge $E_{1}$ and $\hat{s}=1$ at edge $E_{2}$;

- $\hat{s}$ is the normalized arclength along edges $E_{3}$ and $E_{4}$;

- $\hat{t}=0$ at edge $E_{3}$ and $\hat{t}=1$ at edge $E_{4}$;

- $\hat{t}$ is the normalized arclength along edges $E_{1}$ and $E_{2}$.

An algebraic transformation $\hat{s}: \mathcal{C} \rightarrow \mathcal{P}$ is defined to map the computation space $\mathcal{C}$ onto the parameter space $\mathcal{P}$ :

$$
\begin{aligned}
& \hat{s}=\hat{s}_{E_{3}}(\xi)(1-\hat{t})+\hat{s}_{E_{4}}(\xi) \hat{t}, \\
& \hat{t}=\hat{t}_{E_{1}}(\eta)(1-\hat{s})+\hat{t}_{E_{2}}(\eta) \hat{s},
\end{aligned}
$$

where $\hat{s}_{E_{3}}$ is the normalized arc length along edge $E_{3}$, etc.

The elliptic transformation $x: \mathcal{P} \rightarrow \mathcal{D}$ is defined to map the parameter space $\mathcal{P}$ onto the physical domain $\mathcal{D}$. In the physical domain, the curvilinear coordinate system satisfies a system of Laplace equations

$$
\Delta r=0,
$$

where $r=(x, y)^{T}$. Then the Laplace system, Equation (12), is transformed to the computational space $\mathcal{C}$ giving

$$
g^{11} r_{\xi \xi}+2 g^{12} r_{\xi \eta}+g^{22} r_{\eta \eta}+\Delta \xi r_{\xi}+\Delta \eta r_{\eta}=0,
$$


where

$$
\begin{aligned}
& {\left[\begin{array}{c}
\Delta \xi \\
\Delta \eta
\end{array}\right]=g^{11} P_{11}+2 g^{12} P_{12}+g^{22} P_{22},} \\
& g^{11}=g_{22} / J^{2}=\left(r_{\eta}, r_{\eta}\right) / J^{2}, \quad g^{12}=-g_{12} / J^{2}=-\left(r_{\xi}, r_{\eta}\right) / J^{2}, \\
& g^{22}=g_{11} / J^{2}=\left(r_{\xi}, r_{\xi}\right) / J^{2}, \quad P_{11}=\left[\begin{array}{c}
P_{11}^{(1)} \\
P_{11}^{2}
\end{array}\right]=-T^{-1}\left[\begin{array}{c}
\hat{s}_{\xi \xi} \\
\hat{t}_{\xi \xi}
\end{array}\right], \\
& P_{12}=\left[\begin{array}{c}
P_{12}^{(1)} \\
P_{12}^{2}
\end{array}\right]=-T^{-1}\left[\begin{array}{c}
\hat{s}_{\xi \eta} \\
\hat{t}_{\xi \eta}
\end{array}\right], \quad P_{22}=\left[\begin{array}{c}
P_{22}^{(1)} \\
P_{22}^{2}
\end{array}\right]=-T^{-1}\left[\begin{array}{c}
\hat{s}_{\eta \eta} \\
\hat{t}_{\eta \eta}
\end{array}\right],
\end{aligned}
$$

and the matrix

$$
\Gamma=\left[\begin{array}{cc}
\hat{s}_{\xi} & \hat{s}_{\eta} \\
\hat{t}_{\xi} & \hat{t}_{\eta}
\end{array}\right]
$$

Note that $r$ and $P$ are vectors and $g$ is a matrix. The details can be found in [16].

\subsection{High-order compact schemes}

Traditional finite difference schemes are based on Lagrange interpolation and require one grid point more than the order of the finite difference scheme; for example, a second order scheme needs to use three grid points. A Padetype compact scheme could be constructed based on Hermite interpolation where both function and derivatives at grid points are involved, for example, a fourth order finite difference scheme can be constructed if both function and first order derivative are used at three grid points. For a function $f$ we may write a compact scheme using five points [14]:

$$
\begin{aligned}
& \beta_{-} f_{j-2}^{\prime}+\alpha_{-} f_{j-1}^{\prime}+f_{j}^{\prime}+\alpha_{+} f_{j+1}^{\prime}+\beta_{+} f_{j+2}^{\prime} \\
& =\left(b_{-} f_{j-2}+a_{-} f_{j-1}+c f_{j}+a_{+} f_{j+1}+b_{+} f_{j+2}\right) / \Delta \xi .
\end{aligned}
$$

We can get eighth order of accuracy by using the above formula. To obtain a symmetric and tri-diagonal system, we may set $\beta_{-}=\beta_{+}=0, \alpha_{-}=\alpha_{+}=\frac{1}{3}$, 


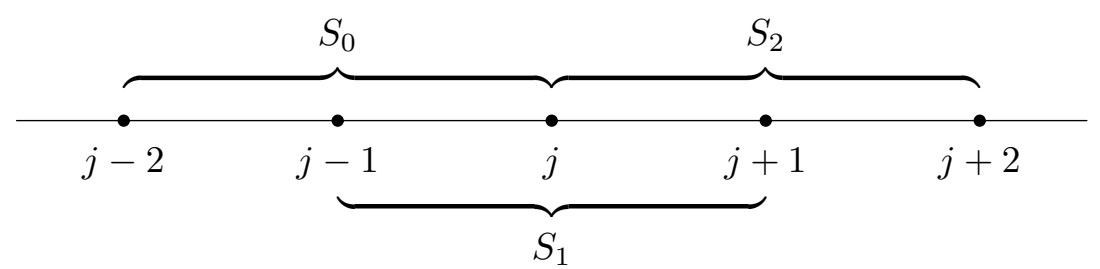

Figure 2: Candidate stencils for an interior point $j$.

$a_{+}=-a_{-}=\frac{7}{9}, b_{+}=-b_{-}=\frac{1}{36}, c=0$, giving a sixth order scheme that uses three derivatives and five grid points.

\subsection{Weighted compact scheme}

If the compact scheme is used to differentiate a discontinuous or shock function, the computed derivative has grid to grid oscillation. In previous work [9], we proposed a new class of finite difference scheme - the weighted compact scheme (WCS). This scheme not only achieves high order accuracy and high resolution, but also accurately captures shock waves without oscillation.

\subsubsection{Basic formulations of weighted compact scheme}

For a given point $j$, three candidate stencils containing this point are (see Figure 2)

$$
S_{0}=\left(x_{j-2}, x_{j-1}, x_{j}\right), \quad S_{1}=\left(x_{j-1}, x_{j}, x_{j+1}\right) \quad \text { and } \quad S_{2}=\left(x_{j}, x_{j+1}, x_{j+2}\right) .
$$

The schemes for the three candidate stencils are obtained by applying Equation (17) to each of these stencils:

$$
S_{0}: \quad F_{0}: \quad 2 f_{j-1}^{\prime}+f_{j}^{\prime}=\frac{1}{h}\left(-\frac{1}{2} f_{j-2}-2 f_{j-1}+\frac{5}{2} f_{j}\right),
$$




$$
\begin{array}{lll}
S_{1}: & F_{1}: & \frac{1}{4} f_{j-1}^{\prime}+f_{j}^{\prime}+\frac{1}{4} f_{j+1}^{\prime}=\frac{1}{h}\left(-\frac{3}{4} f_{j-1}+\frac{3}{4} f_{j+1}\right), \\
S_{2}: & F_{2}: & 2 f_{j+1}^{\prime}+f_{j}^{\prime}=\frac{1}{h}\left(-\frac{5}{2} f_{j}+2 f_{j+1}+\frac{1}{2} f_{j+2}\right) .
\end{array}
$$

The schemes corresponding to stencils $S_{0}$ and $S_{2}$ are third order, one sided, finite difference schemes; and the scheme corresponding to $S_{1}$ is a fourth order, centered scheme. These three equations are denoted by $F_{0}, F_{1}$ and $F_{2}$. A new scheme is obtained using the weighted average

$$
F=C_{0} F_{0}+C_{1} F_{1}+C_{2} F_{2},
$$

where $C_{0}, C_{1}$ and $C_{2}$ are weights that satisfy $C_{0}+C_{1}+C_{2}=1$. When the weights are chosen as

$$
C_{0}=C_{2}=\frac{1}{18}, \quad C_{1}=\frac{8}{9},
$$

the resulting scheme is the sixth order centered compact scheme

$$
\frac{1}{3} f_{j-1}^{\prime}+f_{j}^{\prime}+\frac{1}{3} f_{j+1}^{\prime}=\frac{1}{h}\left(-\frac{1}{36} f_{j-2}-\frac{7}{9} f_{j-1}+\frac{7}{9} f_{j+1}+\frac{1}{36} f_{j+2}\right) .
$$

The procedure described above shows that a sixth order centered compact scheme can be represented by a combination of three lower order schemes. In order to achieve the non-oscillatory property, the WENO weights [7] are introduced to determine each stencil. The weights are determined according to the smoothness of the function on each stencil. Following the WENO method, the new weights are

$$
\omega_{k}=\frac{\gamma_{k}}{\sum_{i=0}^{2} \gamma_{i}} \quad \text { and } \quad \gamma_{k}=\frac{C_{k}}{\left(\varepsilon+I S_{k}\right)^{\sigma}},
$$

where $\varepsilon$ is a small positive number used to prevent the denominator becoming zero, $\sigma$ is an important parameter to control the weighting and $I S_{k}$ are smoothness measurements:

$$
I S_{0}=\frac{13}{12}\left(f_{j-2}-2 f_{j-1}+f_{j}\right)^{2}+\frac{1}{4}\left(f_{j-2}-4 f_{j-1}+3 f_{j}\right)^{2},
$$




$$
\begin{aligned}
& I S_{1}=\frac{13}{12}\left(f_{j-1}-2 f_{j}+f_{j+1}\right)^{2}+\frac{1}{4}\left(f_{j-1}-f_{j+1}\right)^{2} \\
& I S_{2}=\frac{13}{12}\left(f_{j}-2 f_{j+1}+f_{j+2}\right)^{2}+\frac{1}{4}\left(f_{j}-4 f_{j+1}+3 f_{j+2}\right)^{2} .
\end{aligned}
$$

Regard the two terms on the right hand side as the measurements of the curvature and the slope respectively at a certain point.

The new scheme is

$$
F=\omega_{0} F_{0}+\omega_{1} F_{1}+\omega_{2} F_{2} .
$$

The leading error of $F$ is a combination of the leading errors of the original schemes, and is

$$
\left(\frac{1}{12} \omega_{0}-\frac{1}{12} \omega_{2}\right) f^{(4)} h^{3}+\left(-\frac{1}{15} \omega_{0}+\frac{1}{120} \omega_{1}-\frac{1}{15} \omega_{2}\right) f^{(5)} h^{4} .
$$

\subsubsection{Conservation and reconstruction function}

The conservative property of the scheme is very important in shock wave capturing. Conservation can be obtained when the weighted compact scheme is applied together with the ENO [5] reconstruction method [9].

\subsection{Linear stability (CFL conditions) for stretched grids}

\subsubsection{CFL condition}

Consider the 1D advection equation

$$
\frac{\partial u}{\partial t}+a \frac{\partial u}{\partial x}=0 \quad(-\infty<x<+\infty) \text { such that } u(0, x)=\sin (k x),
$$


where $a$ and $k$ are constants. The Euler forward and up-winding scheme gives, for $a>0$, the finite difference scheme

$$
w_{i}^{n+1}=w_{i}^{n}+a \frac{\Delta t}{\Delta x}\left(w_{i}^{n}-w_{i-1}^{n}\right), \quad w_{i}^{0}=\sin \left(k x_{i}\right) .
$$

Here $w_{i}^{n}$ is an approximation of the exact solution at $x=x_{i}$ and $t=t_{n}$. This difference equation is stable if $\left|a \frac{\Delta t}{\Delta x}\right| \leq 1$ which means the CFL number must be less than or equal to one. The exact solution is $u=\sin k(x-a t)$.

\subsubsection{CFL condition for stretched and curved grids}

For a 3D inviscid compressible flow, the governing Euler equation in general curvilinear coordinates is

$$
\frac{\partial Q}{\partial \tau}+\frac{\partial F}{\partial \xi}+\frac{\partial G}{\partial \eta}+\frac{\partial H}{\partial \zeta}=0
$$

where $\xi=\xi(x, y, z), \eta=\eta(x, y, z), \quad \zeta=\zeta(x, y, z), \tau=t$ and $Q=J[\rho, \rho u, \rho v, \rho w, e]^{T}$. Here $J$ is the Jacobian, $\rho$ is the density, $u, v$ and $w$ are velocity components in $x, y$ and $z$ directions respectively, and $e$ is the internal energy. For a 1D hyperbolic system in the $\xi$ direction, equation (28) diagonalizes to

$$
\frac{\partial q}{\partial \tau}+\Lambda \frac{\partial q}{\partial \xi}=S
$$

where $\Lambda$ is a diagonal matrix with the five eigenvalues

$$
\begin{aligned}
\lambda_{\xi}^{1}=\lambda_{\xi}^{2}=\lambda_{\xi}^{3} & =\xi_{x} u+\xi_{y} v+\xi_{z} w=U, \\
\lambda_{\xi}^{4} & =U+c\left(\xi_{x}^{2}+\xi_{y}^{2}+\xi_{z}^{2}\right)^{\frac{1}{2}}, \\
\lambda_{\xi}^{5} & =U-c\left(\xi_{x}^{2}+\xi_{y}^{2}+\xi_{z}^{2}\right)^{\frac{1}{2}},
\end{aligned}
$$

where $U$ is the contra-variant velocity component and $c=\sqrt{\gamma R T_{r}}$ is the speed of sound. The non-dimensional eigenvalues are

$$
\bar{\lambda}_{\xi}^{1}=\bar{\lambda}_{\xi}^{2}=\bar{\lambda}_{\xi}^{3}=\bar{U},
$$




$$
\begin{aligned}
\bar{\lambda}_{\xi}^{4} & =\bar{U}+\frac{1}{M_{r}}\left(\xi_{x}^{2}+\xi_{y}^{2}+\xi_{z}^{2}\right)^{\frac{1}{2}}, \\
\bar{\lambda}_{\xi}^{5} & =\bar{U}-\frac{1}{M_{r}}\left(\xi_{x}^{2}+\xi_{y}^{2}+\xi_{z}^{2}\right)^{\frac{1}{2}},
\end{aligned}
$$

where $U=\bar{U} / U_{r}$ and $M_{r}=U_{r} / c$ is the Mach number. The CFL condition requires $\left|\lambda_{\xi}^{k} \Delta t / \Delta \xi\right| \leq 1$. If $M_{r}$ is too small (low speed flow), $\lambda_{\xi}^{4}$ or $\lambda_{\xi}^{5}$ will be extremely large even when $U$ is near zero close to the wall, in which case $\Delta t$ must be extremely small. In the formulas above, $\xi_{x} \approx \Delta \xi / \Delta x$ represents the stretching, $\xi_{y} \approx \Delta \xi / \Delta y$ and $\xi_{z} \approx \Delta \xi / \Delta z$ represent the curvature. Note that $\Delta \xi$ is usually constant ( $\Delta \xi=$ domain length/grid number), and $\lambda_{\xi}^{4}$ or $\lambda_{\xi}^{5}$ will be extremely large if $\Delta x, \Delta y, \Delta z$ are too small or the stretching or distortion is too big. It is always recommended to check the size of $\max \left[\xi_{x}, \xi_{y}, \xi_{z}\right] / \min \left[\xi_{x}, \xi_{y}, \xi_{z}\right]$ and make sure that it is not too large.

Using implicit marching is a nice alternative, but we should be careful to talk about the accuracy of the implicit scheme when the nonlinear system is not fully converged at each time step.

\subsection{Non-linearity and high-order filter}

\subsubsection{Non-linearity and high-frequency waves}

Now consider the non-linear advection equation

$$
\frac{\partial u}{\partial t}+u \frac{\partial u}{\partial x}=0 \quad(-\infty<x<+\infty) \quad \text { such that } u(0, x)=\sin (k x) .
$$

The Euler forward scheme would give

$$
\begin{aligned}
w_{i}^{1} & =w_{i}^{1}+\left.\Delta t w_{i}^{1} \frac{\partial w}{\partial x}\right|_{i} ^{1}=\sin \left(k x_{i}\right)+\Delta t \sin \left(k x_{i}\right) \cos \left(k x_{i}\right) \\
& =\sin \left(k x_{i}\right)+\Delta t \sin \left(2 k x_{i}\right) / 2, \\
w_{i}^{0} & =\sin \left(k x_{i}\right) .
\end{aligned}
$$


The initial solution originally does not include the wave number $2 k$. However, after the first step, the solution has both wave number $k$ and wave number $2 k$. As time increases, the maximum wave number will become larger and larger due to progressive doubling until it cannot be resolved by our computational grid. Consequently, we will have problems with the grid resolution when we solve the non-linear advection equation.

\subsubsection{Low passing filter}

This problem with the grid resolution can be solved using a filter which significantly reduces the high-frequency waves. Recently the following high order implicit filter has been developed and widely applied [14, 19]:

$$
\alpha \hat{\varphi}_{i-1}+\hat{\varphi}_{i}+\alpha \hat{\varphi}_{i+1}=\sum_{n=0}^{N} \frac{a_{n}}{2}\left(\varphi_{i+n}+\varphi_{i-n}\right),
$$

where $2 N$ is the number of neighbouring points, $-0.5<\alpha<0.5, \hat{\varphi}_{i}$ is filtered, $\varphi_{i}$ is the original value and the $a_{n}$ are coefficients for neighboring points. Note that LES uses a filter for the governing equation, but the filter discussed here is to filter the solution itself. In any case, the filtered part of the solution or equation should be tracked by using a sub-scale model.

\subsubsection{Filter and artificial viscosity}

The artificial viscosity concept is to add additional dissipation to the NavierStokes equation to reduce the effective Reynolds number. For example, the solution of a second order finite difference approximation of the incompressible Navier-Stokes equations will satisfy

$$
\frac{\partial u_{i}}{\partial t}+\frac{\partial u_{i} u_{j}}{\partial x_{j}}-\left(\frac{1}{\operatorname{Re}}+d h^{2}\right) \frac{\partial^{2} u_{i}}{\partial x_{j}^{2}}+\frac{\partial p}{\partial x_{i}}=0
$$


where $h$ is the mesh size and $d$ is a constant which is directly related to the finite difference scheme. The filter discussed above only allows low frequency waves to pass, damping the high-frequency waves. There are no governing equations involved in the filter. However, if we substitute the original $\varphi$ by the filtered $\hat{\varphi}$ in the governing equations, we find we really add some dissipation. In general,

$$
u_{i}=\hat{u}_{i}+O\left(h^{k}\right) .
$$

Replace $u$ by the above formula in the governing Navier-Stokes equation, we change the governing equation and possibly add some dissipation. However, if the filter has higher order, we do not change the governing equation in the numerical sense.

\subsection{Fully implicit scheme and iteration (flow solver)}

In Equation (1), a second order backward scheme is used for time derivative, and the fully implicit form of the discretized equation is

$$
\begin{aligned}
& \frac{3 Q^{n+1}-4 Q^{n}+Q^{n-1}}{2 J \Delta t}+\frac{\partial\left(E^{n+1}-E_{v}^{n+1}\right)}{\partial \xi} \\
& \quad+\frac{\partial\left(F^{n+1}-F_{v}^{n+1}\right)}{\partial \eta}+\frac{\partial\left(G^{n+1}-G_{v}^{n+1}\right)}{\partial \zeta}=0 .
\end{aligned}
$$

The value $Q^{n+1}$ is estimated iteratively as $Q^{n+1}=Q^{p}+\delta Q^{p}$ with $Q^{0}=Q^{n}$. The flux vectors are linearized as $E^{n+1} \approx E^{P}+A^{P} \delta Q^{P}, F^{n+1} \approx F^{P}+B^{P} \delta Q^{P}$ and $G^{n+1} \approx G^{P}+C^{P} \delta Q^{P}$, so that equation (37) becomes

$$
\left[\frac{3}{2} I+\Delta t J\left(D_{\xi} A+D_{\eta} B+D_{\zeta} C\right)\right] \delta Q^{P}=\text { Res },
$$

where the residual

$$
\text { Res }=-\left(\frac{3}{2} Q^{p}-2 Q^{n}+\frac{1}{2} Q^{n-1}\right)
$$




$$
-\Delta t J\left[D_{\xi}\left(E-E_{v}\right)+D_{\eta}\left(F-F_{v}\right)+D_{\zeta}\left(G-G_{v}\right)\right]^{p} .
$$

Here $D_{\xi}, D_{\eta}$ and $D_{\zeta}$ represent partial differential operators, and

$$
A=\frac{\partial E}{\partial Q}, \quad B=\frac{\partial F}{\partial Q}, \quad G=\frac{\partial G}{\partial Q}
$$

are the Jacobian matrices of flux vectors. The right hand side of Equation (38) is discretized using a sixth order, compact scheme for the spatial derivatives, and the equation is solved by LU-SGS method [21]. As $\delta Q^{p}$ is driven to zero, $Q^{p}$ approaches $Q^{n+1}$.

An eighth order compact filter is employed at each time step to reduce numerical oscillation.

\subsection{Non reflecting boundary conditions}

The concept of non-reflecting boundary conditions was proposed by Thompson $[17,18]$ who introduced the idea of specifying the boundary conditions according to the inward and outward propagating waves. Usually the outgoing waves have their behaviour defined entirely by the solution at and within the boundary, and no boundary conditions are needed. Therefore, the number of boundary conditions equals the number of incoming waves. Poinsot and Lele [15] extended Thompson's method to specify the boundary conditions for the Navier-Stokes equations, where the effect of viscosity has been taken into account. However, only boundary conditions in Cartesian coordinates are given. Based on the previous work by the above authors, we developed non-reflecting boundary conditions for compressible flow in curvilinear coordinates [10]. Based on a 1D characteristic analysis, the hyperbolic terms in $\xi$ direction is modified to

$$
\frac{\partial \rho}{\partial t}+d_{1}+V \frac{\partial \rho}{\partial \eta}+\rho\left(\eta_{x} \frac{\partial u}{\partial \eta}+\eta_{y} \frac{\partial v}{\partial \eta}+\eta_{z} \frac{\partial w}{\partial \eta}\right)+W \frac{\partial \rho}{\partial \zeta},
$$




$$
\begin{aligned}
+\rho\left(\zeta_{x} \frac{\partial u}{\partial \zeta}+\zeta_{y} \frac{\partial v}{\partial \zeta}+\zeta_{z} \frac{\partial w}{\partial \zeta}\right)+\text { vis }_{1} & =0 \\
\frac{\partial u}{\partial t}+d_{2}+V \frac{\partial u}{\partial \eta}+\frac{1}{\rho} \eta_{x} \frac{\partial p}{\partial \eta}+W \frac{\partial u}{\partial \zeta}+\frac{1}{\rho} \zeta_{x} \frac{\partial p}{\partial \zeta}+v_{i s} & =0 \\
\frac{\partial v}{\partial t}+d_{3}+V \frac{\partial v}{\partial \eta}+\frac{1}{\rho} \eta_{y} \frac{\partial p}{\partial \eta}+W \frac{\partial v}{\partial \zeta}+\frac{1}{\rho} \zeta_{y} \frac{\partial p}{\partial \zeta}+v_{3} & =0 \\
\frac{\partial w}{\partial t}+d_{4}+V \frac{\partial w}{\partial \eta}+\frac{1}{\rho} \eta_{z} \frac{\partial p}{\partial \eta}+W \frac{\partial w}{\partial \zeta}+\frac{1}{\rho} \zeta_{z} \frac{\partial p}{\partial \zeta}+\operatorname{vis}_{4} & =0 \\
\frac{\partial p}{\partial t}+d_{5}+V \frac{\partial p}{\partial \eta}+\gamma p\left(\eta_{x} \frac{\partial u}{\partial \eta}+\eta_{y} \frac{\partial v}{\partial \eta}+\eta_{z} \frac{\partial w}{\partial \eta}\right)+W \frac{\partial \rho}{\partial \zeta} & 0 \\
+\gamma p\left(\zeta_{x} \frac{\partial u}{\partial \zeta}+\zeta_{y} \frac{\partial v}{\partial \zeta}+\zeta_{z} \frac{\partial w}{\partial \zeta}\right)+v i s_{5} & =0
\end{aligned}
$$

where vis $_{1}-$ vis $_{5}$ represent viscous terms in curvilinear coordinates, and

$$
\left[\begin{array}{l}
d_{1} \\
d_{2} \\
d_{3} \\
d_{4} \\
d_{5}
\end{array}\right]=\left[\begin{array}{c}
\frac{1}{c^{2}}\left[\frac{1}{2}\left(L_{1}+L_{5}\right)+L_{2}\right] \\
\frac{\xi_{x}}{2 \beta \rho c}\left(L_{5}-L_{1}\right)-\frac{1}{\beta^{2}}\left(\xi_{y} L_{3}+\xi_{z} L_{4}\right) \\
\frac{\xi_{y}}{2 \beta \rho c}\left(L_{5}-L_{1}\right)+\frac{1}{\beta^{2} \xi_{x}}\left[\left(\xi_{x}^{2}+\xi_{z}^{2}\right) L_{3}-\xi_{z} \xi_{y} L_{4}\right] \\
\frac{\xi_{z}}{2 \beta \rho c}\left(L_{5}-L_{1}\right)-\frac{1}{\beta^{2} \xi_{x}}\left[\xi_{y} \xi_{z} L_{3}-\left(\xi_{x}^{2}+\xi_{y}^{2}\right) L_{4}\right] \\
\frac{1}{2}\left(L_{1}+L_{5}\right)
\end{array}\right]
$$

In Equation (42), $c$ is the sound wave speed and $\beta=\sqrt{\xi_{x}^{2}+\xi_{y}^{2}+\xi_{z}^{2}}$. The $L_{i}$ represent the amplitude variations of the characteristic waves corresponding to the characteristic velocities, which are $\lambda_{1}=U-C_{\xi}, \lambda_{2}=\lambda_{3}=\lambda_{4}=U$ and $\lambda_{5}=U+C_{\xi}$, where $C_{\xi}=c \beta$. The amplitude variations are

$$
\begin{aligned}
L_{1} & =\left(U-C_{\xi}\right)\left[-\frac{\rho c}{\beta}\left(\xi_{x} \frac{\partial u}{\partial \xi}+\xi_{y} \frac{\partial v}{\partial \xi}+\xi_{z} \frac{\partial w}{\partial \xi}\right)+\frac{\partial p}{\partial \xi}\right] \\
L_{2} & =U\left(c^{2} \frac{\partial \rho}{\partial \xi}-\frac{\partial p}{\partial \xi}\right), \\
L_{3} & =U\left(-\xi_{y} \frac{\partial u}{\partial \xi}+\xi_{x} \frac{\partial v}{\partial \xi}\right),
\end{aligned}
$$




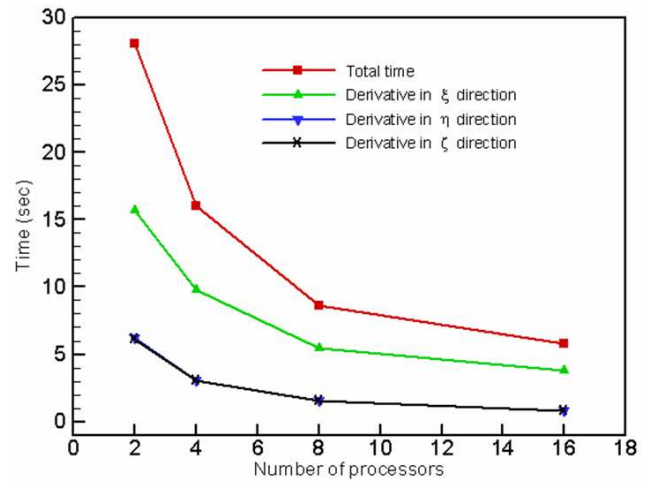

(a)

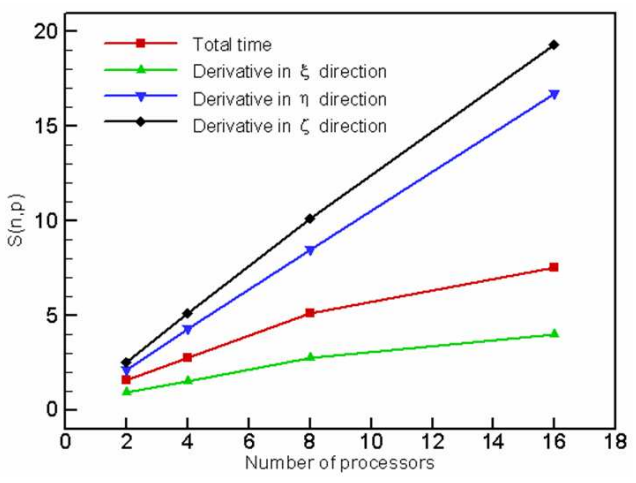

(b)

FigURE 3: Linear speed-up of MPI computation: (a) Wall-clock time versus number of processors; (b) speed-up versus number of processors.

$$
\begin{aligned}
L_{4} & =U\left(-\xi_{z} \frac{\partial u}{\partial \xi}+\xi_{x} \frac{\partial w}{\partial \xi}\right) \\
L_{5} & =\left(U+C_{\xi}\right)\left[\frac{\rho c}{\beta}\left(\xi_{x} \frac{\partial u}{\partial \xi}+\xi_{y} \frac{\partial v}{\partial \xi}+\xi_{z} \frac{\partial w}{\partial \xi}\right)+\frac{\partial p}{\partial \xi}\right] .
\end{aligned}
$$

These equations will be used for neighbours of boundary points in the $\xi$ direction. The equations for $\eta$ and $\zeta$ directions are similar. In this way, the non-physical wave reflection is effectively eliminated.

\subsection{MPI parallel computation}

We developed an MPI parallel code based on our serial code. The performance of the parallel program is examined for our compressible DNS code on an SGI Origin 2000 computer and the results show very good computing efficiency of parallel machines with MPI (see Figure 3). 


\section{DNS for flow transition}

\subsection{Problem definitions and boundary conditions}

Nonlinear stability, starting with the formation of three dimensional structures, is also referred to as secondary instability. The aligned three dimensional structure associated with the peak valley splitting of secondary instability was first measured in detail by Klebanov, Tidstrom and Sargent [12]. This type of secondary instability is now referred to as the fundamental or Ktype after Klebanov. Later, in boundary layer experiments, Kachanov [11] found another type of secondary instability characterized by subharmonic signals and that reveals staggered patterns of three dimensional structure. These experiments showed the staggered structure of unstable vortices, which is referred to as H-type after Herbert [6].

The computation domain is displayed in Figure 4. The length of computational domain along the streamwise direction is about 32 primary TS wavelengths which amounts to around $800 \delta_{\text {in }}$, where $\delta_{\text {in }}$ is defined as the displacement thickness of inflow boundary layer. Here, the TS wave refers to Tollmien-Schlichting wave which is the most unstable mode for flow instability on a flat plate. The width along the spanwise direction is about $30 \delta_{\text {in }}$, and height at the inflow boundary is $40 \delta_{\text {in }}$. The Reynolds number at the inflow is 1000 based on the displacement thickness and the free-stream velocity. The Mach number is set to 0.5 .

The inflow boundary conditions are in the form

$$
q=q_{\mathrm{lam}}+A_{2 d} q_{2 d}^{\prime}+A_{3 d} q_{3 d}^{\prime}+A_{r} q_{r}^{\prime},
$$

where $q$ stands for the velocity components, the pressure, and the density. The two dimensional Blasius-like profile obtained from the solution of the similarity equation is denoted by $q_{\mathrm{lam}}, q_{2 d}^{\prime}$ represents the eigenmode of two dimensional Tollmien-Schlichting (TS) waves with a space wave number of 


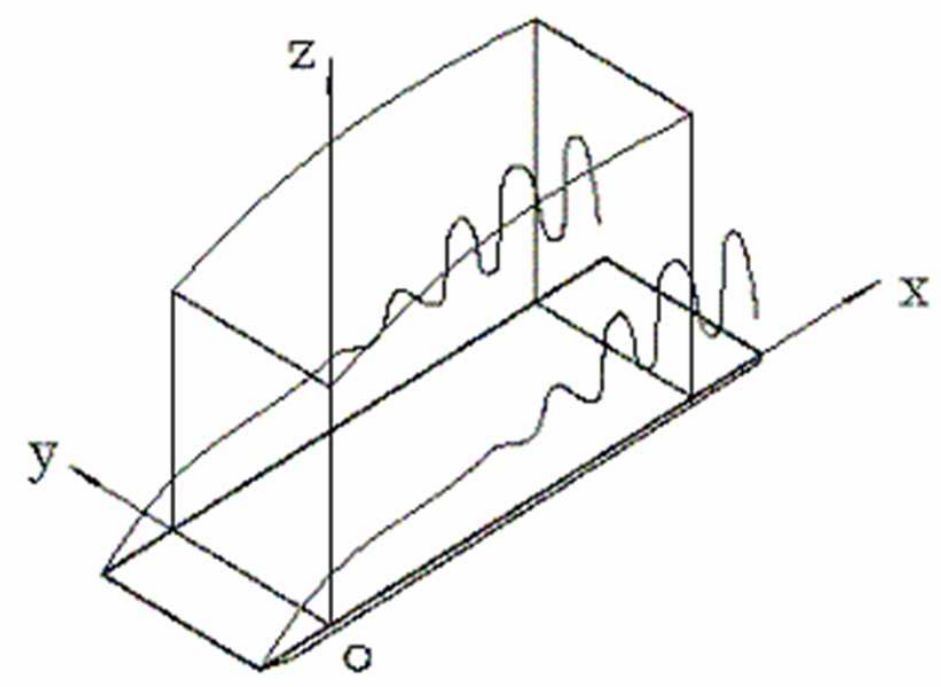

Figure 4: Computation domain.

$\alpha_{2 d}=\alpha_{2 d}^{r}+i \alpha_{2 d}^{i}$ and a frequency of $\omega_{2 d}$, and $q_{3 d}^{\prime}$ denotes the eigenmode of three dimensional TS waves with a space wave number $\alpha_{3 d}=\alpha_{3 d}^{r}+i \alpha_{3 d}^{i}$ and $\tilde{\beta}$, which is defined as an angle between the wave and mean flow directions, and a frequency of $\omega_{3 d}$. The disturbance associated with a random white noise is denoted by $q_{r}^{\prime}$ which changes from -1 to 1 randomly. The amplitude of the two dimensional, three dimensional and random noise are denoted by $A_{2 d}$, $A_{3 d}$, and $A_{r}$ respectively.

In order to understand the transition, we ran several simulation cases in the smaller grids of $768 \times 64 \times 80$. All simulations were carried out on SGI Origin 3900 computers using up to 16 processors. In the following the type of transition observed is indicated by an $\mathrm{H}$ or $\mathrm{K}[1,13]$. 


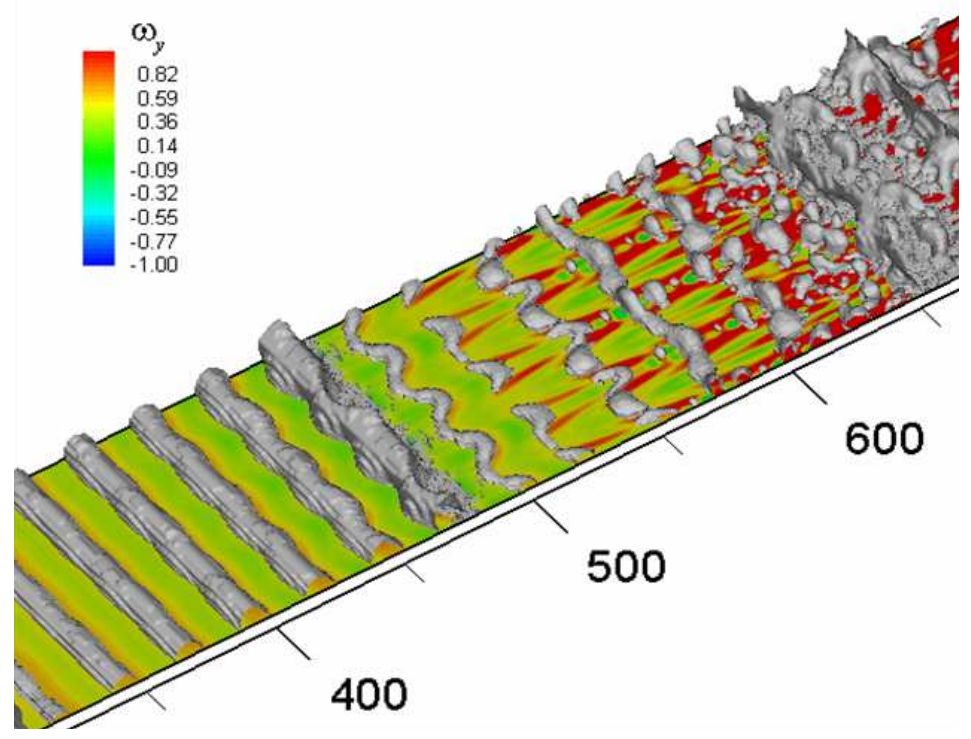

FiguRE 5: Iso-surface of spanwise vorticity for the K-Type transition. 


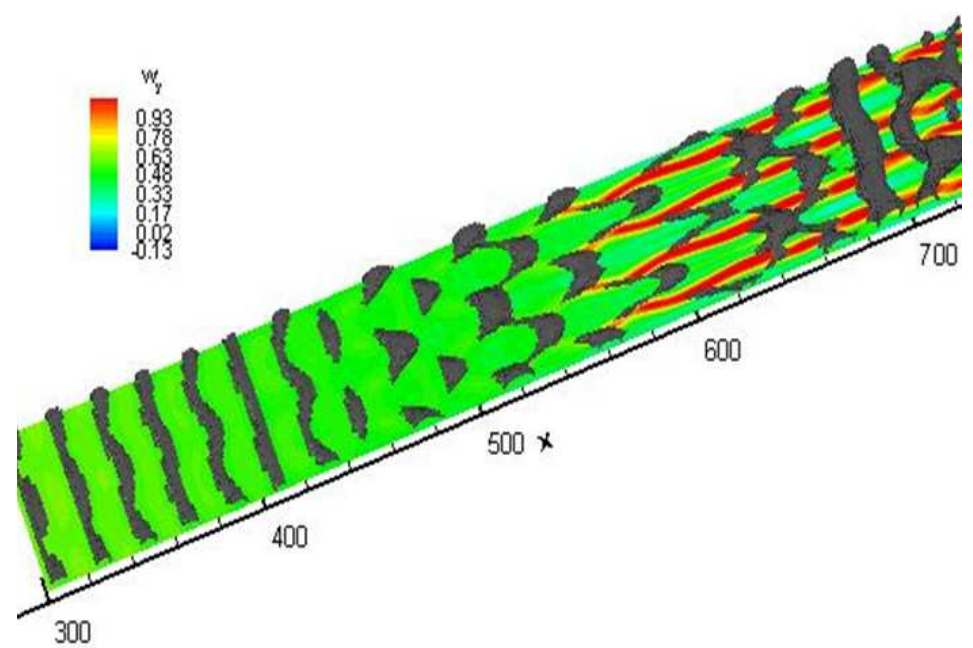

Figure 6: Iso-surface of spanwise vorticity for the H-Type transition.

\subsection{Computational results}

The K-type transition is shown in Figure 5 and H-type in Figure 6. Figure 7 shows the skin friction coefficient calculated from the time-averaged and spanwise-averaged velocity profile. The skin friction coefficient of the laminar flow is plotted as a dashed line and the turbulent boundary layer as a dash-dotted line. The $C_{f}$ curve from the simulation coincides with the laminar flow curve before $x=500 \delta_{\text {in }}$. A sudden growth in skin friction occurs after $x=500 \delta_{\text {in }}$ indicating the transition from laminar to turbulent flow. The post-transition $C_{f}$ level is comparable to that of fully developed turbulent flow. The fluctuation of during and after the transition represents the unsteadiness of turbulent flow and the DNS results show good agreement between numerical and experimental results when compared for laminar (dashed line) and turbulent (dash-dotted line) flow. This shows that the validation of DNS is achieved (Ducros, [4]) for the case of flow transition over a flat plate. 


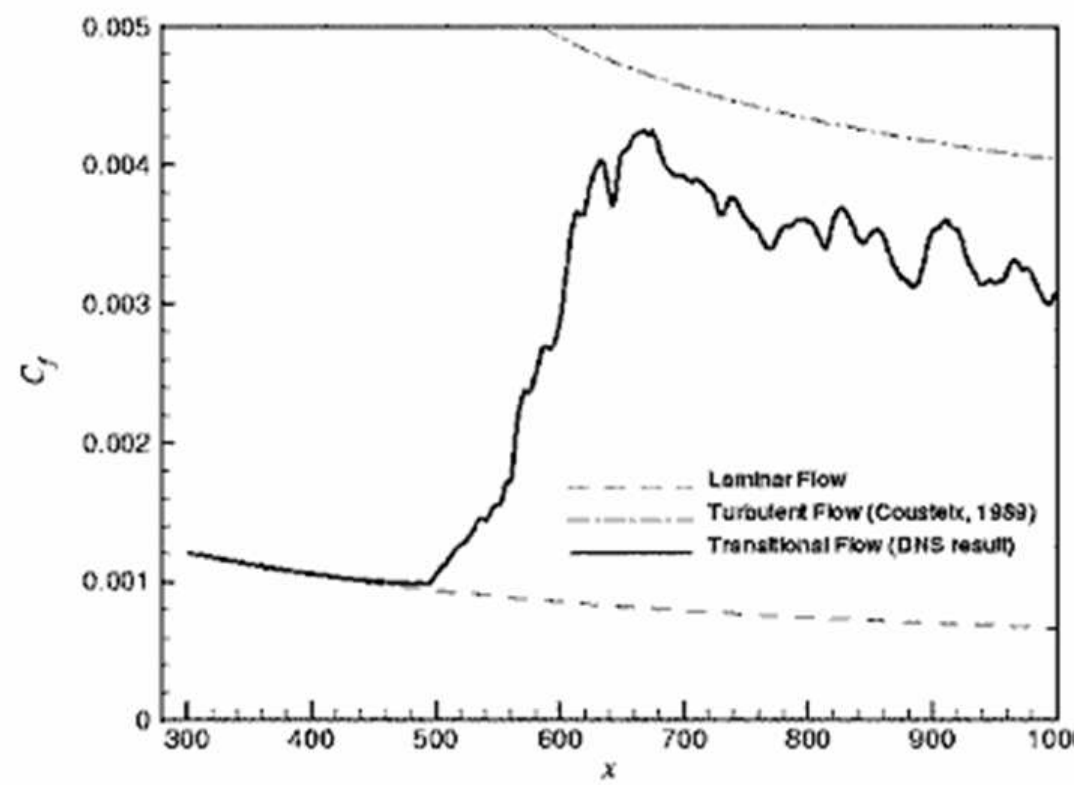

FIGURE 7: Time and spanwise averaged skin-friction coefficient. 


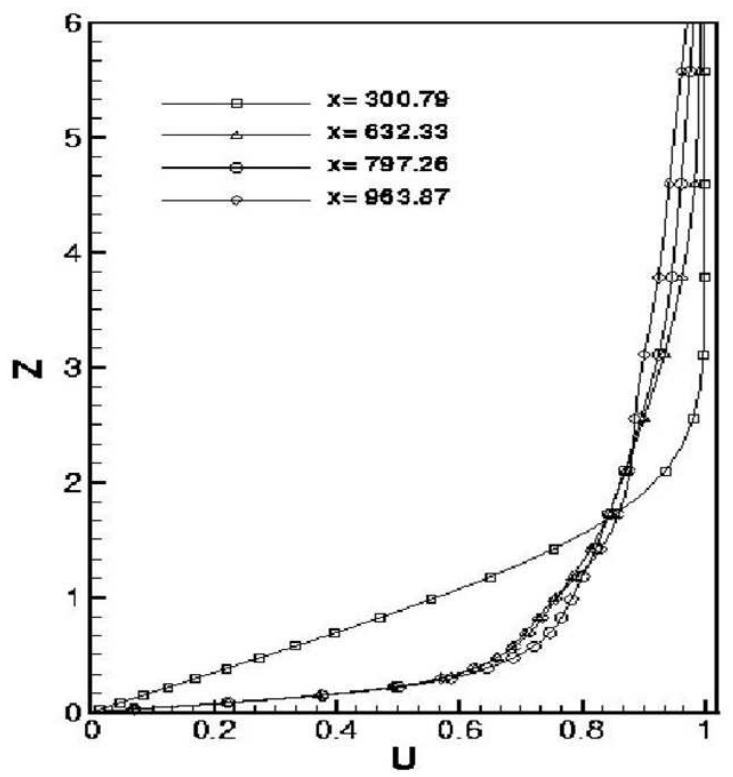

FiguRE 8: Time and spanwise averaged velocity profiles along the plate. 
The time-averaged and spanwise-averaged streamwise velocity profiles after nine periods of time for various streamwise locations are displayed in Figure 8 . The inflow velocity profile at $x=300.79 \delta_{\text {in }}$ is of a typical laminar flow. At $x=627.9 \delta_{\text {in }}$, the profile starts to change, and the velocity profiles clearly become turbulent after $x=800$. The log law of the streamwise velocity is observed.

This DNS code has been validated by NASA Langley researchers for many different cases related to flow transition including flat plate, cones and swept wings [8].

\section{DNS for flow separation and control around an airfoil}

\subsection{Problem definitions and boundary conditions}

Numerical simulations are performed for a NACA0012 airfoil at an attack angle of $4^{\circ}$. The free stream velocity $U_{r}$, pressure $p_{r}$, temperature $T_{r}$ and chord length of the airfoil $C$ are selected as the reference velocity, pressure, temperature and length respectively, and are used to non-dimensionalize the governing equations. The computational domain is plotted in Figure 9. The upstream boundary is three chord lengths away from the leading edge of the airfoil. The upper and lower boundaries are about four chord lengths from the surface. The outflow boundary is two chord lengths downstream of the trailing edge. The airfoil is regarded as infinite in the spanwise direction. In our simulation, the spanwise length is set as $L_{y}=0.1 C$, and a periodic boundary condition is imposed at the spanwise boundaries.

The flow and computational conditions are listed in Table 1. Here $\Delta x^{+}$, $\Delta y^{+}$and $\Delta z^{+}$are the mesh sizes scaled by the shear stress on the wall surface. Grid distributions in the $(x, z)$ plane and on the airfoil surface are shown in 


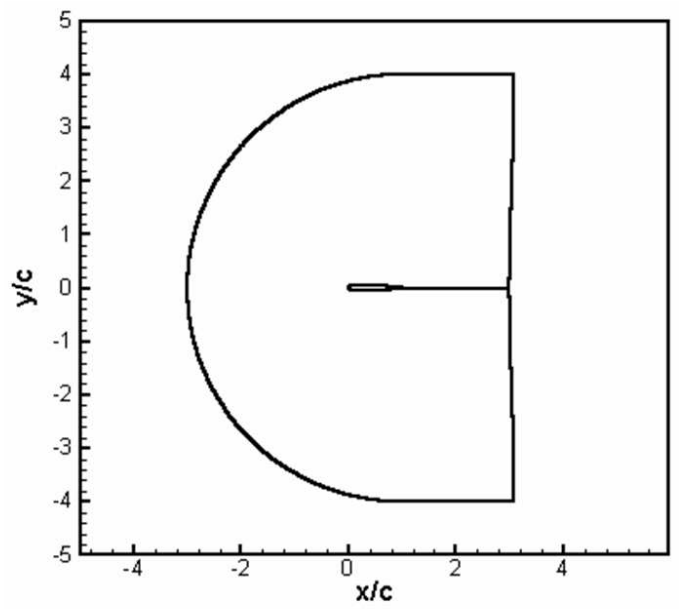

Figure 9: Computational domain.

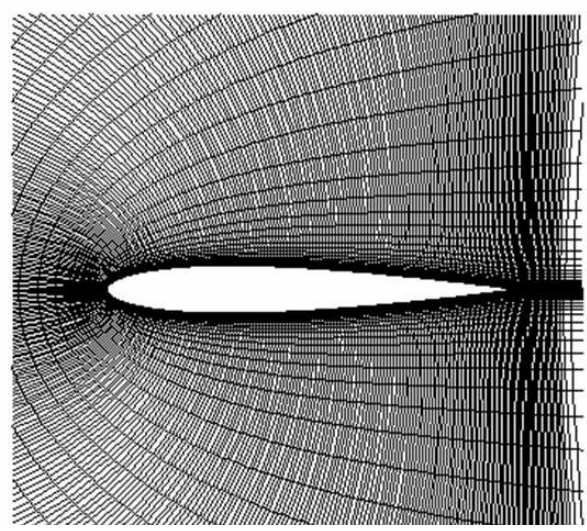

(a)

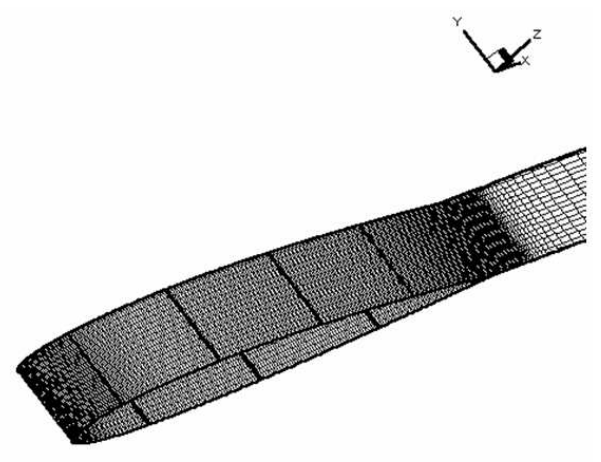

(b)

Figure 10: Grid distribution: (a) in $(x, z)$ plane, and (b) on the airfoil surface. 


$$
\begin{array}{ccccccc}
\text { TABLE 1: } & \text { Flow and computational conditions. } \\
\operatorname{Re}=U_{r} C / \nu & M_{r} & A O A & N_{x} \times N_{y} \times N_{z} & \Delta x^{+} & \Delta y^{+} & \Delta z^{+} \\
10^{5} & 0.2 & 4^{\circ} & 1200 \times 32 \times 180 & <13 & <15 & <1
\end{array}
$$

Figure 10. The computational domain is divided evenly into $M$ sub-domains along the $\xi$ direction when $M$ processors are used. In this work, 24 processors are used for all cases.

\subsection{Computational results and analysis}

Three cases were considered:

4.2.1 Baseline case without blowing;

4.2.2 Pulsed blowing;

4.2.3 Blowing jet with a $30^{\circ}$ pitch angle and a $90^{\circ}$ skew angle.

All simulations were carried out with a time step equal to $8.35 \times 10^{-5} \mathrm{C} / U_{r}$. The corresponding CFL number is around 400.

\subsubsection{Flow around the airfoil without blowing (baseline case)}

Flow separation and vortex shedding appear on the suction surface of the airfoil (Figure 11), where a separated mixing layer and vortex shedding are clearly demonstrated by plotting contours of instantaneous spanwise vorticity. The separation zone can be seen clearly from the time averaged velocity vectors shown in Figure 12. There is no vortex breakdown observed in the $2 \mathrm{D}$ simulation since the breakdown is a $3 \mathrm{D}$ and non-linear interaction. 


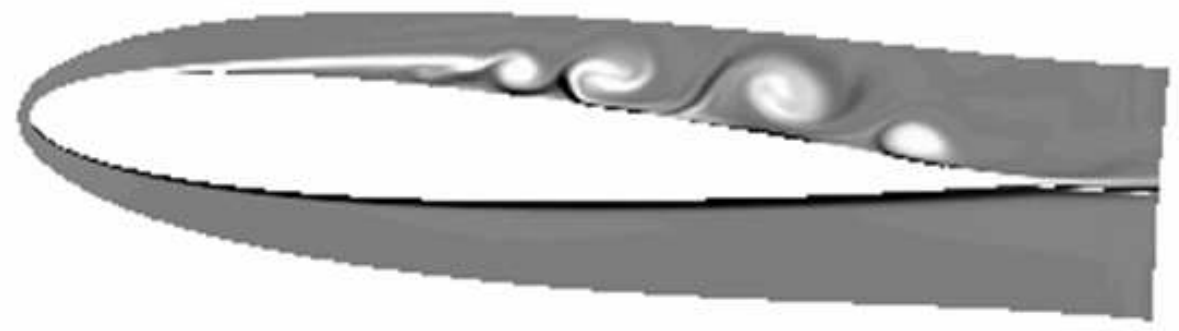

Figure 11: Contours of spanwise vorticity from 2D solution.

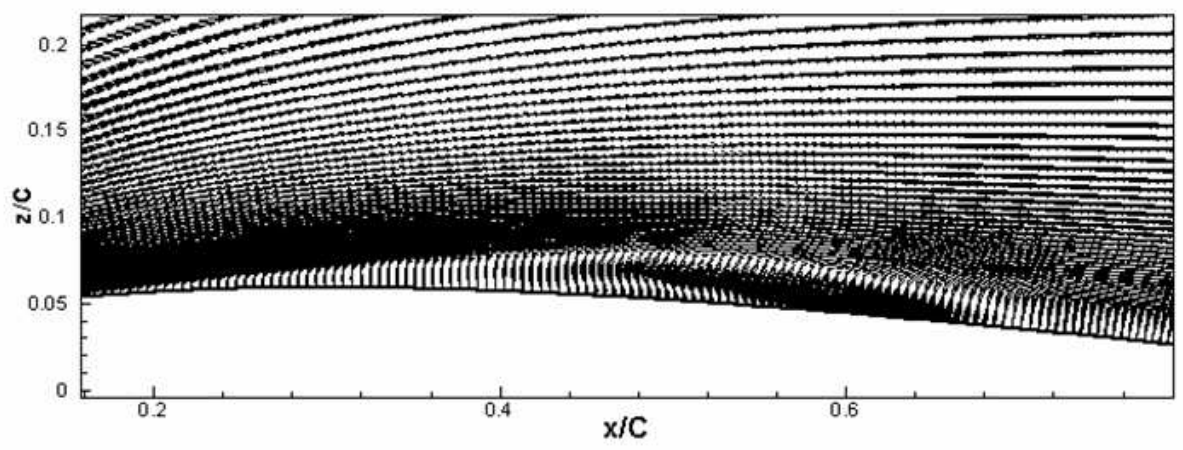

FiguRE 12: Time averaged velocity field. 


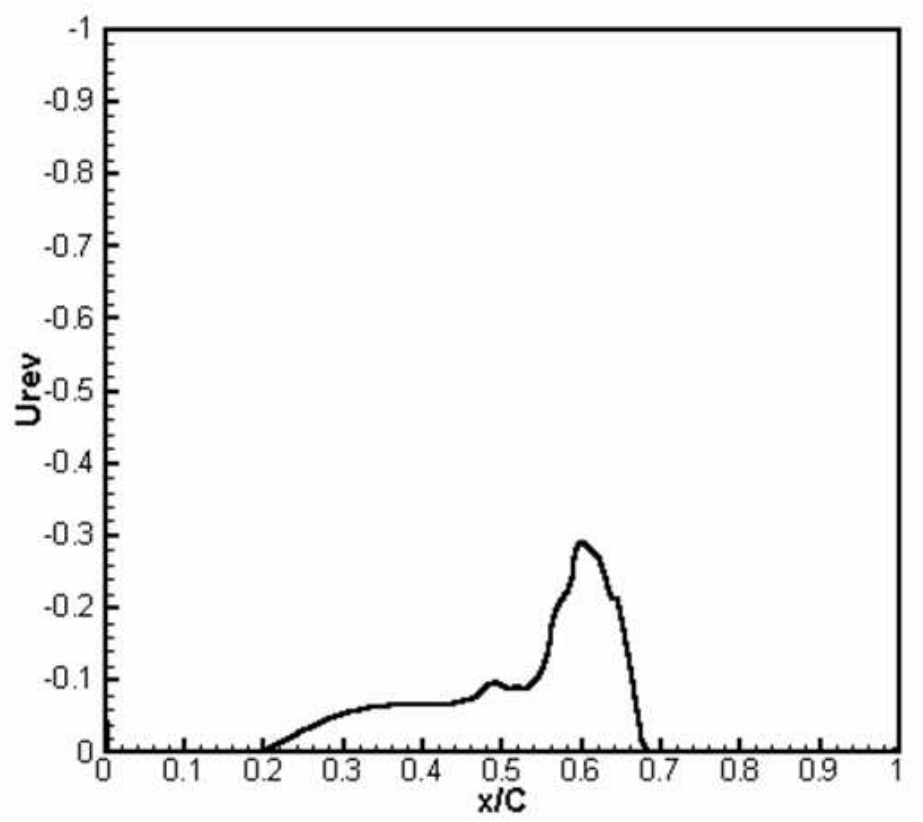

Figure 13: Reverse flow distribution on the suction side $\left(U_{\mathrm{rev}}=\min (u)\right)$.

Three dimensional solutions are highly unsteady. Figure 13 shows the maximum reverse flow in the wall normal direction along the suction surface. The separated zone appears from $x / C=0.19$ to $x / C=0.68$, where the separated laminar boundary evolves into reattached turbulent boundary layer. The reverse flow reaches $8 \%$ of the free stream velocity at about $x / C=0.5$, then increases to just less than $30 \%$ of the free stream velocity at about $x / C=0.6$ before rapidly falling to zero at $x / C=0.68$.

The iso-surfaces of instantaneous vorticity in the spanwise direction are plotted in Figure 14. The transition process and breakdown of the rolling-up shear layer are clearly demonstrated. The vortices shed from the separated shear layer are distorted while travelling downstream. The interactions of 3D structures cause the spanwise vorticity iso-surface to break into small pieces, 


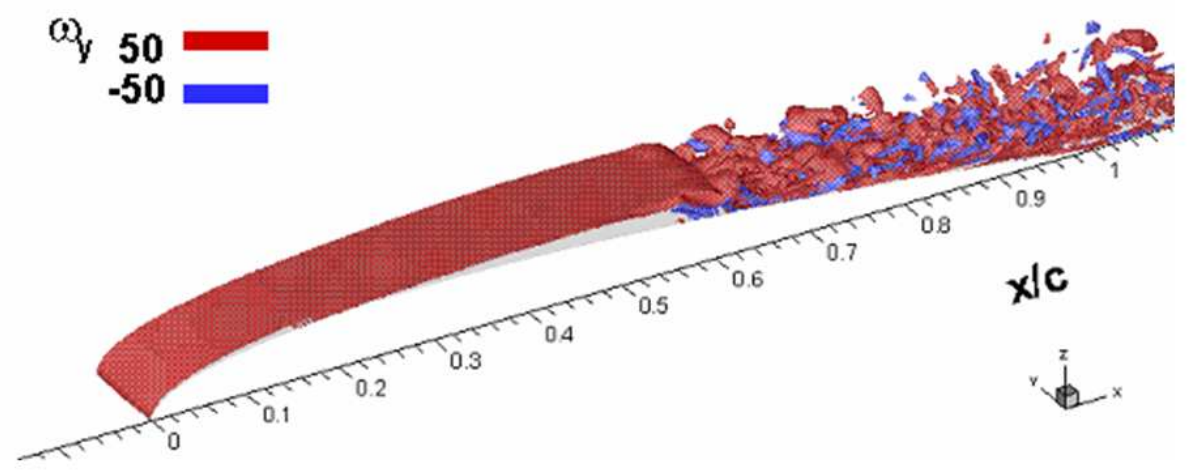

FIGURE 14: Iso-surface of instantaneous spanwise vorticity.

indicating that vortex breakdown occurs. The boundary layer becomes fully turbulent after reattachment.

We did not add any perturbation at inflow to trigger the Kelvin-Helmhotz instability, but our spectrum analysis shows that the flow instability starts from the wake and, thus, we believe that unsteady wakes produce the initial disturbance which propagates upstream due to the parabolic feature in time and elliptic feature in space of the Navier-Stokes equations. The three dimensional instability that emerges in the simulation originates from the near wake region, where intensive interactions between the large-scale vortical structure and the wake occur. The three dimensional instability seems to be self sustained and leads to the transition to turbulence.

\subsubsection{Flow around the airfoil with a pulsed blowing jet}

Unsteady blowing [2] is enforced from $x_{0}=0.153$ to $x_{1}=0.175$, which is before the separation point $x_{s}=0.19$. The non-dimensional frequency of blowing $F^{+}=F C / U_{r}$, where $C$ is the chord length and $F$ is the frequency, is set to be 2 (we now believe 1.4 is a better choice). With this configuration, 

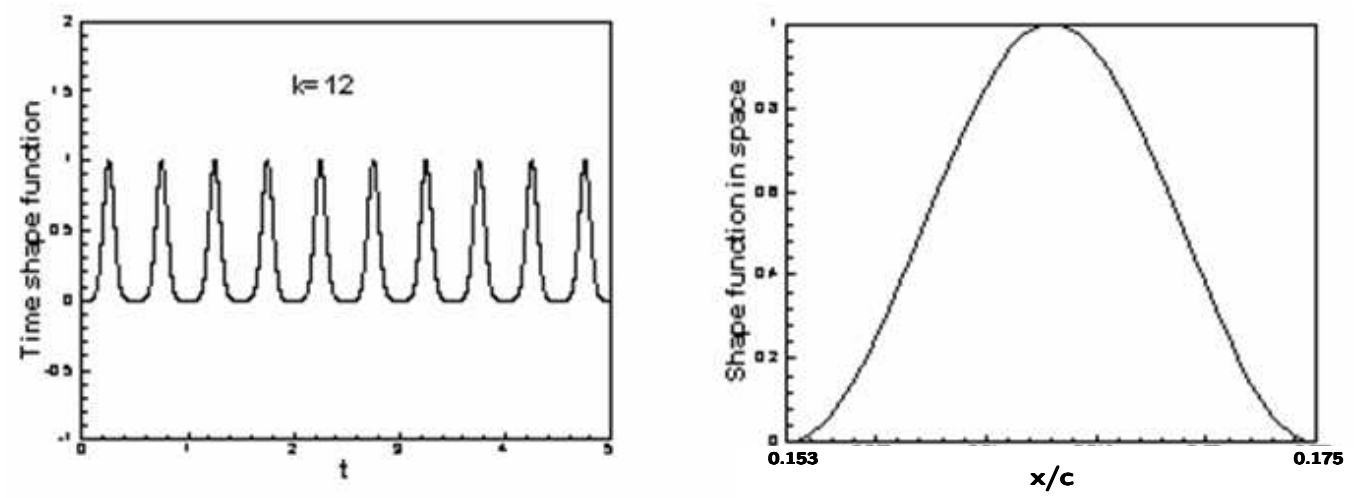

FIGURE 15: Shape functions in time and space.

the blowing velocity is directed in the wall-normal direction and has the magnitude, for $x \in\left[x_{0}, x_{1}\right]$,

$$
w(x, y, t)=A\left(0.5-0.5 \cos \theta_{x}\right)\left(0.5-0.5 \cos \theta_{y}\right) \exp \left[-k\left(\frac{2 \tilde{\tau}}{\tilde{T}}-1\right)^{2}\right],
$$

where $\theta_{x}=2 \pi\left(x-x_{0}\right) /\left(x_{1}-x_{0}\right), \theta_{y}=2 \pi y / L_{y}, \tilde{\tau}=t-n \tilde{T}$ where $n$ is the integer part of $\tilde{\tau} / \tilde{T}$, and $\tilde{T}=1 / F^{+}$. The spatial distribution and temporal variation of the blowing velocity are depicted in Figure 15 . The values $A=$ 0.4 and $k=12$ were used in this case; these parameters control the blowing mass rate.

The time integration for unsteady blowing case reached $t=3.73 C / U_{\infty}$. Time averaging is performed only over three periods of blowing due to the high cost of DNS. More periods are required to get more accurate results. Mean velocity vectors are shown in Figure 16. It is obvious that large separation zone which is clearly seen in the baseline case shown in Figure 16(a) is dramatically reduced (almost removed, see Figure 16(b)).

The reduced separation zone can also be seen from Figure 17 in which the 


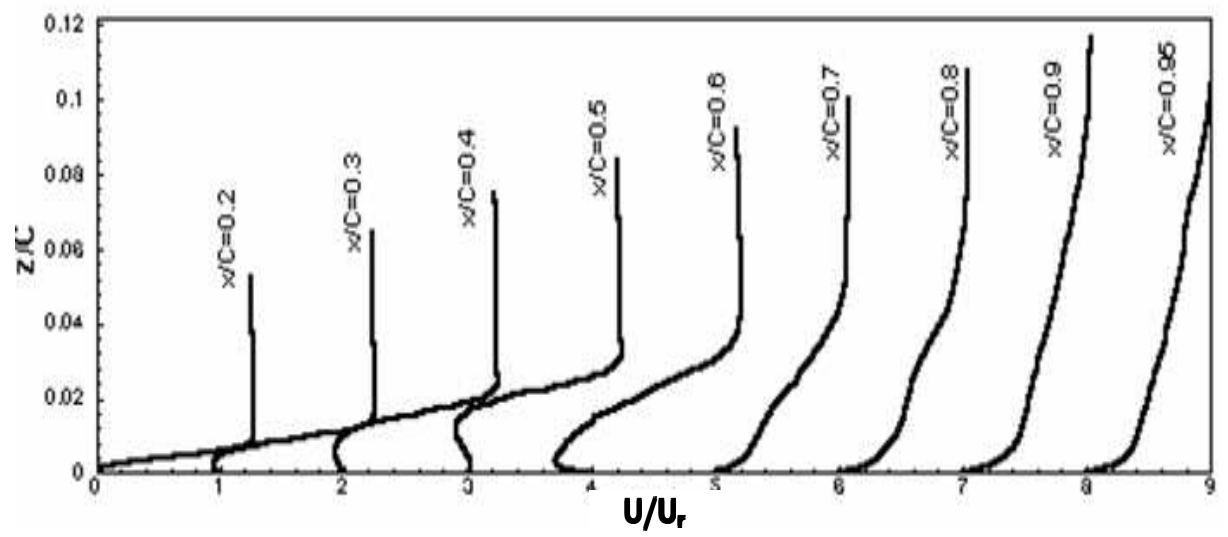

(a)

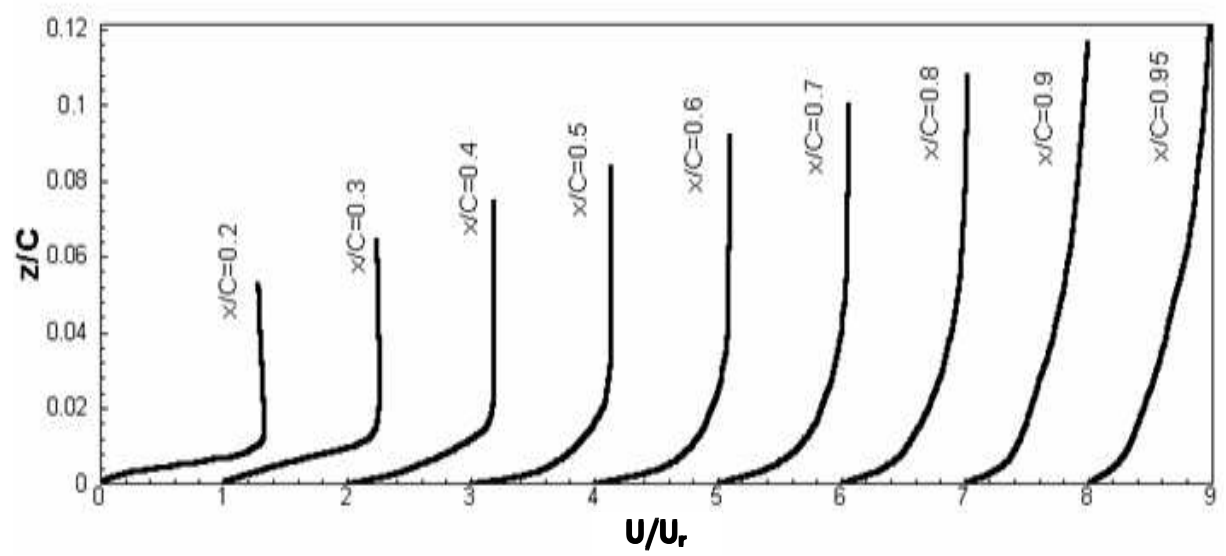

(b)

Figure 16: Streamwise mean velocity profiles for (a) baseline case, and (b) blowing case. 


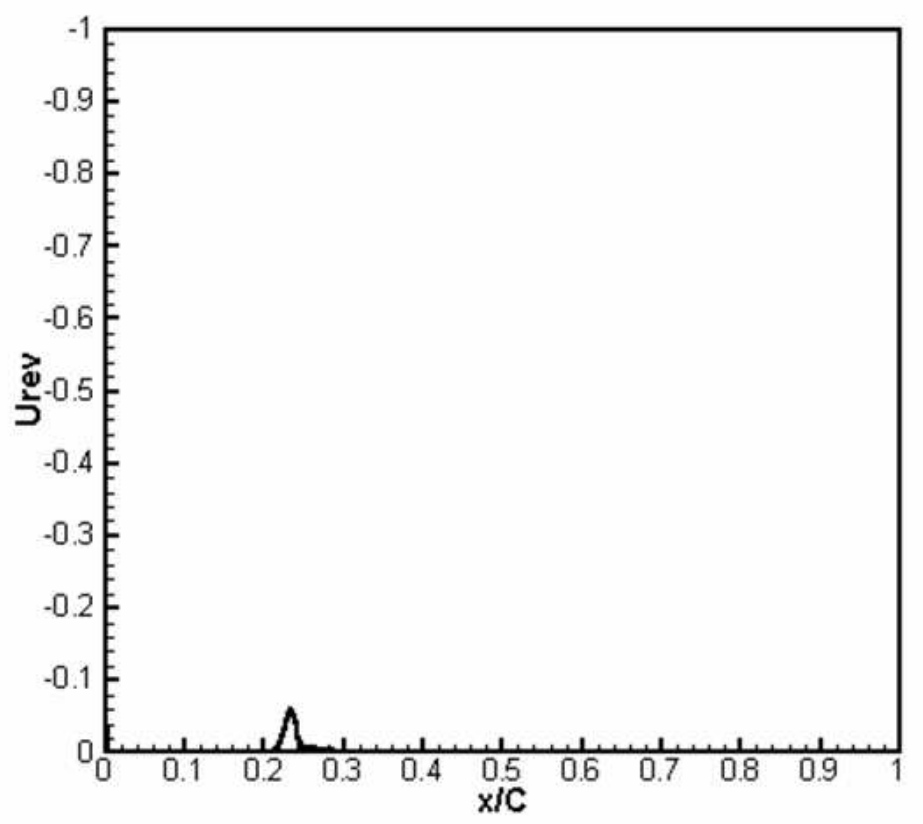

FiguRE 17: Reverse flow distribution on the suction side.

maximum reverse velocity is depicted against streamwise location. Compared with Figure 13, the separation zone is much smaller.

The iso-surfaces of spanwise vorticity components are plotted in Figure 18. The breakdown of the separated shear layer and the development of the vortex structure can be clearly seen. From the simulation results and analysis of this pulsed blowing case, we conclude that properly selected unsteady blowing can trigger the early transition by exciting most unstable waves and non-linear interactions. The blowing can trigger the KelvinHelmoltz instability as well. 


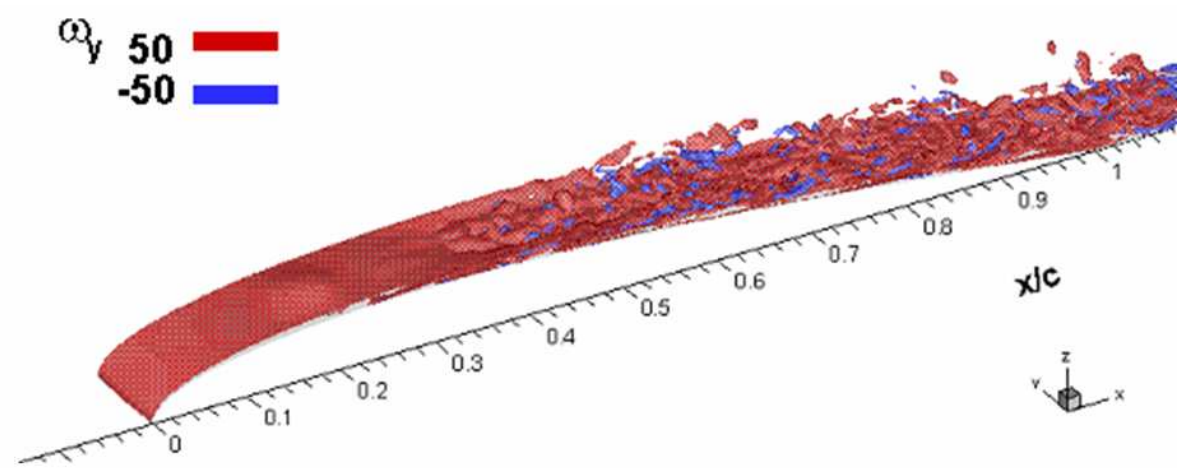

FiguRE 18: Iso-surfaces of spanwise vorticity components.

\subsubsection{The effect of blowing angle}

To study the effect of blowing angle, we set up one case to simulate flow around the airfoil with pulsed blowing of $30^{\circ}$ pitch angle and $90^{\circ}$ skew angles. The pitch angle is defined as an angle between the blowing jet and wall surface. The skew angle is defined as an angle between the blowing jet and the inflow direction. We used the same parameters as before except for $k=300$, which lets the blowing mass be around one fifth of the previous case in Section 4.2.2. The computational results show that the pitched and skewed blowing jets with large $k$ obtained a much better efficiency with a significant increase in the ratio of lift over drag. Temporal variations of lift and drag coefficients which are averaged over the spanwise direction are shown in Figure 19. The pitched jet case has reduced CD and improved CL where $\mathrm{CD}$ and $\mathrm{CL}$ are defined as the coefficients of drag and lift, respectively. The ratio of CL over CD is much improved. The DNS results agree qualitatively with experiments by Bons [2]. 

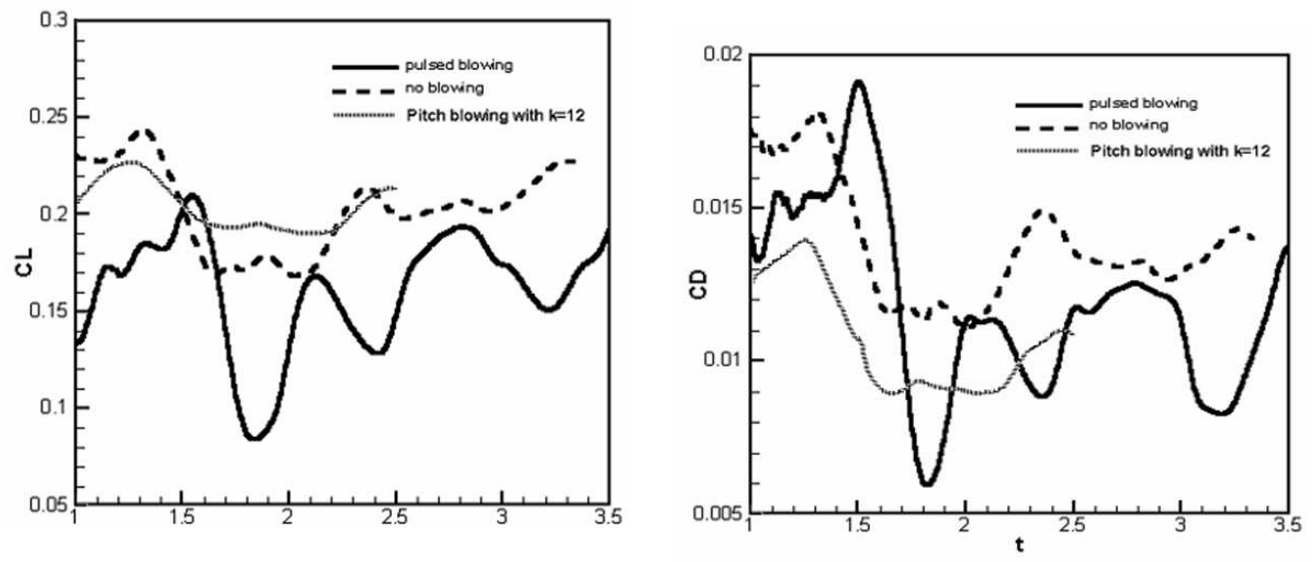

FiguRE 19: Temporal variations of lift and drag coefficients.

\section{LES for wingtip vortex around juncture of wing and flat plate}

\subsection{Grid generation}

In this simulation we concentrate on the tip vortex behind a juncture of wing and flat plate. The physical configuration was simplified by a computational domain, which includes a rectangular wing with a NACA0012 airfoil section and a flat tip, as shown in Figure 20. The span-chord ratio of the wing is 0.75 .

The one-block mesh generation is used in the present work with a single $\mathrm{C}-\mathrm{H}$ topology. Figure 21 shows the C-type grid surrounding the wing on the flat plate. The transformed computational domain (the $\xi-\eta-\zeta$ space) consists of the $\xi$ direction which corresponds to the stream-wise direction, $\eta$ direction which corresponds to the span-wise direction, and $\zeta$ direction which corresponds to the direction normal to the wing surface. The grid for 


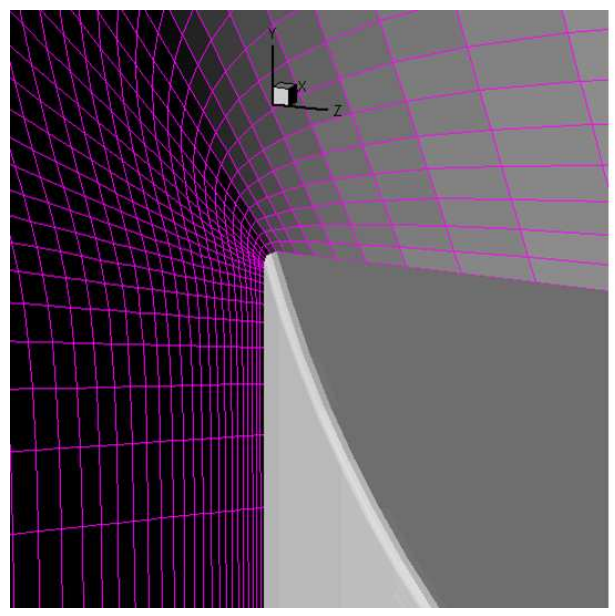

(a)

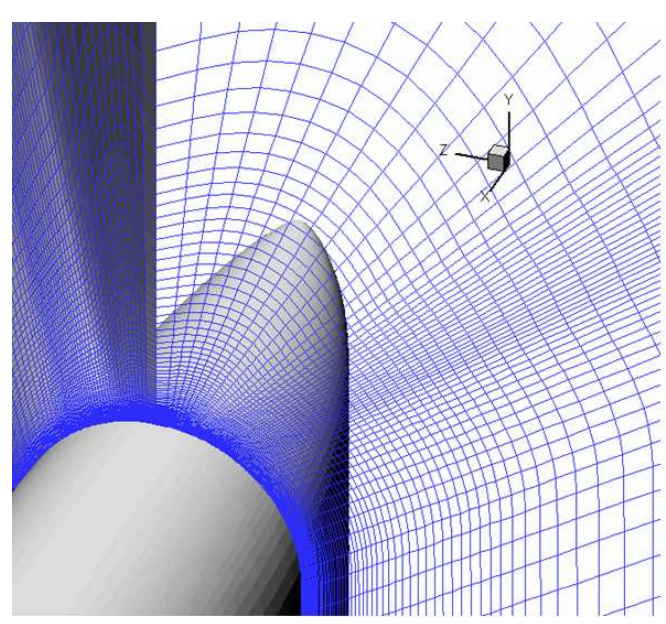

(b)

Figure 20: Grids for (a) the sharp edge, and (b) the rounded edge.

the wing tip is shown in Figure 20.

The computational grid consists of 1536 grid points along the $\xi$ direction, 128 points along the $\eta$ direction and 128 points along the $\zeta$ direction. The whole domain is around $6.0 C$ ( $C$ is the chord length) long, $8.0 C$ wide, and $3.8 C$ high. Figure 21 and Figure 20 show every second grid point and Figure 20 is an enlargement of a local grid.

\subsection{Case setup}

The near-field wakes behind the juncture was simulated with 25 million grid points. The case was set up according to the experiment data given by Chow et al. [3] at $\mathrm{Re}=4.6 \times 10^{6}, M_{r}=0.15$ and an attack angle of $10^{\circ}$. The non-dimensional time step based on the free stream velocity is around $9.0 \times 10^{-5} C / U_{r}$, where $C$ is the chord length and $U_{r}$ is the free stream 


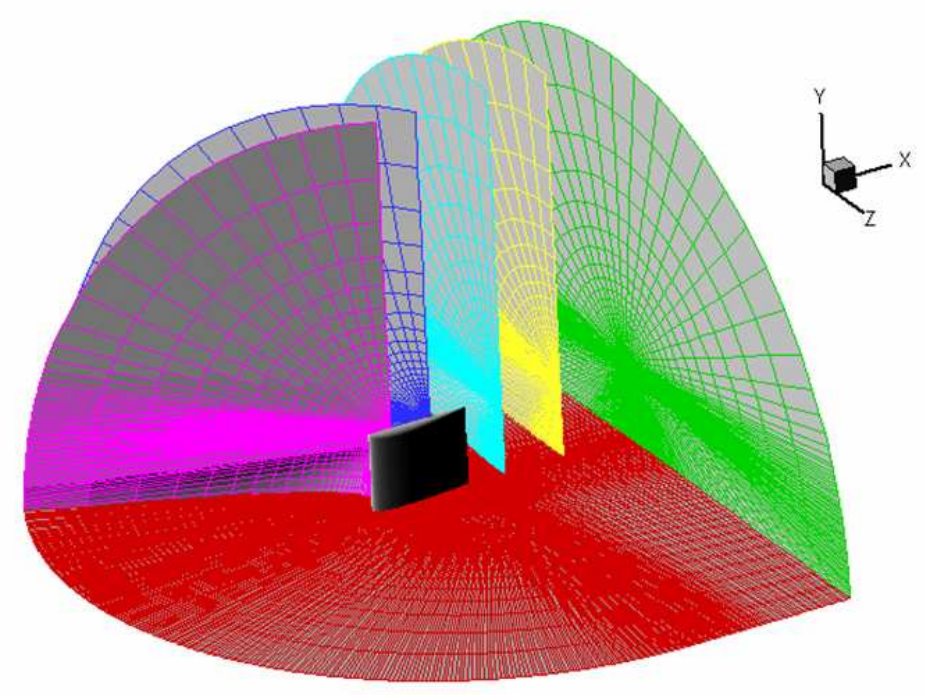

Figure 21: H-C type topology.

velocity. The computation was carried out on 64 CPUs.

\subsection{LES results}

The time-dependent pressure and vorticity contour behind the juncture are depicted in Figure 22 and Figure 23 respectively. Here we chose eight crossflow sections to show the spatial evolution of the tip vortex. The cross-flow sections are chosen at the same locations as Chow et al. [3] used in their experiment so that we can compare the computational and experimental results. The tip vortex is clear and easy to be identified in those contours. The LES results agree qualitatively, but not quantitatively with the experimental results at present. 


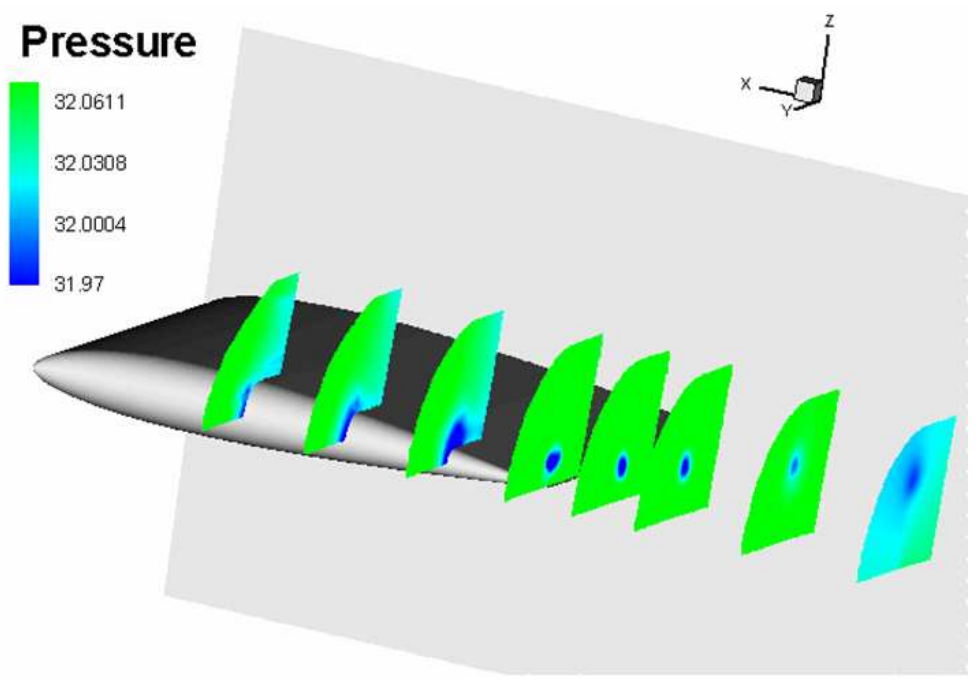

Figure 22: Time-dependent pressure contour.

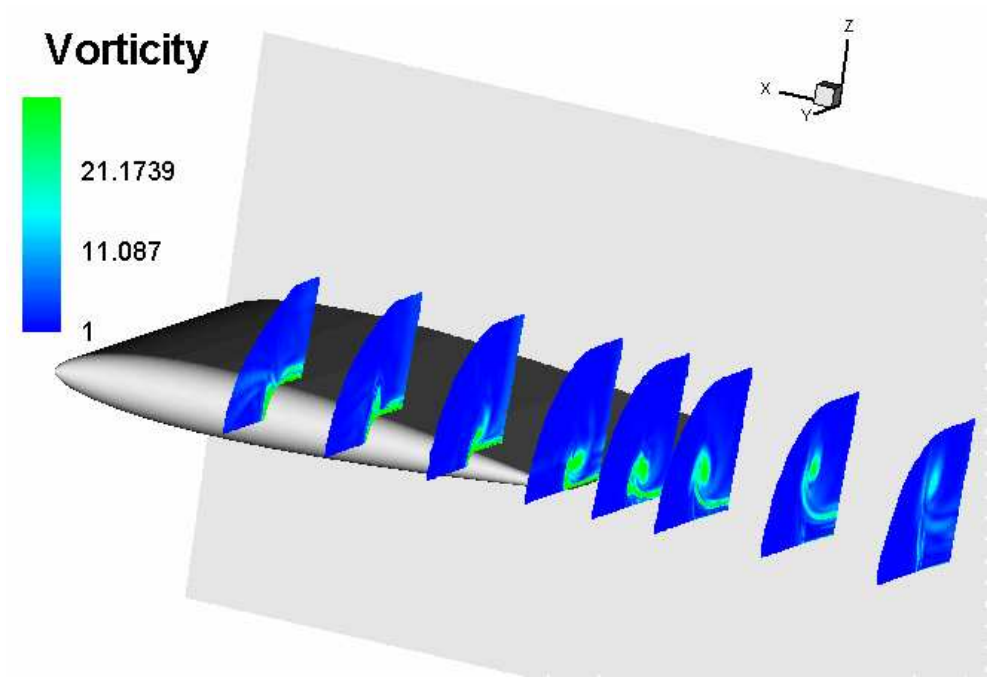

FiguRE 23: Time-dependent vortex contour. 


\section{Conclusions}

- DNS/LES with a high order compact scheme, high order filter, high quality grid generation, and parallel computation can be used for practical engineering applications such as flow instability, separation, transition, flow control and tip vortex.

- DNS can simulate the whole process of flow transition including the $\mathrm{K}$-type and $\mathrm{H}$-type transition and the validation is very good in the friction coefficient and time-averaged and spanwise-averaged velocity profiles (log law).

- Separation and transition processes on a NACA0012 airfoil with or without jet blowing on the surface have been investigated. Though no external disturbances are introduced, the initial perturbations may come from the upward traveling acoustic waves which are generated in the wake. The separated shear layer has an inviscid instability and the perturbation will be quickly amplified at a rate much higher than that of the viscous instability. The traveling disturbances trigger the instability wave which is identified as a Kelvin-Helmholtz instability. The appearance of 3D motions of the shedding primary vortex, where the streamwise vortex appears, and nonlinear interactions of disturbances lead to the sudden growth of disturbances and the generation of high frequencies. The breakdown then happens. The shear layer becomes turbulent and reattaches to the surface.

- Properly selected unsteady blowing triggers early transition by exciting the most unstable waves and non-linear interactions. By selecting appropriate blowing frequencies corresponding to the vortex shedding frequency with 300 pitch angle and 900 skew angle, the separation zone is significantly reduced, and drag is decreased while the lift is maintained at approximately the same level as in the base case. 
- The wing tip vortex can be traced by a high order compact scheme which has very small dissipation.

Acknowledgements: This work was sponsored by the Air Force Office of Scientific Research (AFOSR) and monitored by Dr Len Sakell under grant number F49620-95-1-0018, F49620-97-1-0033, F49620-99-1-0042, F49620-001-0220, Dr Len Sakell and then Tom Beutner F49620-01-1-0028, the NASA Langley Research Center and monitored by Dr Tom Zang and Dr Ron Joslin under grant number NAS1-19016, NAS1-19312, NAG-1-1537, NAG-1-1891, and Dr Meelan Chouderi under sole source purchase order L-14360, L-16516, NNL04AH01P and the Office of Naval Research (ONR) and monitored by Dr Ron Jolsin under grant N00014-03-1-0492. The author also thanks the High Performance Computing Center of US Department of Defense for providing supercomputer hours.

The views and conclusions contained here should not be interpreted as necessarily representing the official policies or endorsements of the AFOSR, NASA, ONR or the US Government.

The work listed in this paper were contributed by a number of my students and post-doctoral researchers including Dr Li Jiang, Dr Hua Shan, Dr Zhining Liu, Dr Sheng Luo, Mr Jiangang Cai, Mr Shutian Deng. Their contributions are sincerely appreciated.

\section{References}

[1] Boiko, A. V., Grek, G. R., Dovgal, A. V., Kozlov, V. V., The origin of turbulence in near-wall flows, pages 167-196, Springer, 2002. C1391 
[2] Bons, J. P., Sondergarrd, R., and Rivir, R. B., Turbine separation control using pulsed vortex generator jets, Journal of Turbomachinery, Vol. 123, pages 198-206, 2001. C1401, C1405

[3] Chow, J. S., Zilliac, G. G., Bradshaw, P., Mean and turbulence mearements in the near field of a wingtip vortex, AIAA J. Vol. 35, 1561-1567, 1997. C1407, C1408

[4] Ducros, F., Comte, P., and Lesieur, M., Large-eddy simulation of transition to turbulence in a boundary layer developing spatially over a flat plate, J. Fluid Mech, Vol. 1, 1996. C1393

[5] Harten, A., Engquist, B., Osher, S., and Chakravarthy, S. R., Uniformly high order accurate essential non-oscillatory schemes, III, Journal of Computational Physics, Vol. 71, pages 231-303, 1987. C1382

[6] Herbert, T., Three-dimensional phenomena in the transitional flat-plate boundary layer, AIAA paper, 1985-0489, 1985. C1390

[7] Jiang, G. S., Shu, C. W., Efficient implementation of weighted ENO scheme, J. Comput. Phys., Vol. 126, pages 202-228, 1996. C1381

[8] Jiang, L., Choudari, M., Chang, C. and Liu, C., Direct numerical simulations of crossflow disturbances in supersonic boundary layers, Journal of Mathematics and Computers in Simulation, Vol. 65, pp 469-487, 2004. C1396

[9] Jiang, L., Shan, H., and Liu, C., Weighted Compact Scheme for Shock Capturing, International Journal of Computational Fluid Dynamics, Vol. 15, pages 147-155, 2001. C1380, C1382

[10] Jiang, L., Shan, H., Liu, C., and Visbal, M., Non-reflecting boundary conditions for DNS in curvilinear coordinates, Recent Advances in DNS and LES, Proceedings of the Second AFOSR (Air Force Office of 
Scientific Research) International Conference, Rutgers, New Jersey, June 7-9, 1999. C1387

[11] Kachanov, Yu. S. and Levchenko, V. Ya., Nonlinear development of a wave in a boundary layer Fluid Dynamics 12, Vol. 383, 1978. C1390

[12] Klebanov, P., Tidstrom, K., and Sargent, L., The three dimensional nature of boundary layer instability, J. Fluid Mech., Vol. 12, 1962. C1390

[13] Liu, C. and Liu, Z., Multigrid mapping and box relaxation for simulation of the whole process of flow transition in 3-D boundary layers, J. of Computational Physics, Vol. 119, pages 325-341, 1995. C1391

[14] Lele, S. K., Compact finite difference schemes with spectral-like resolution, Journal of Computational Physics, Vol. 103, pages 16-42, 1992. C1379, C1385

[15] Poinsot, T. J., Lele, S. K., Boundary conditions for direct simulations of compressible viscous flows, J. Comput. Phys., Vol. 101, pages 104-129, 1992. C1387

[16] Spekreijse, S. P., Elliptic grid generation based on Laplace equations and algebraic transformation, J. Comp. Phys., Vol. 118, pages 38-61, 1995. C1377, C1379

[17] Thompson, K. W., Time dependent boundary conditions for hyperbolic systems, I, J. Comput. Phys., Vol. 68, pages 1-24, 1987. C1387

[18] Thompson, K. W., Time dependent boundary conditions for hyperbolic systems, I, J. Comput. Phys., Vol. 89, pages 439-461, 1990. C1387

[19] Visbal, M. R. and Gaitonde, D. V., High-order accurate methods for unsteady vortical flows on curvilinear meshes, AIAA Paper 98-013, 1998. C1385 
[20] Visbal, M. and Gaitonde, D., On the use of higher-order finite-difference schemes on curvilinear and deforming mashes, J. Comp. Physics, Vol. 181, pages 155-158, 2002. C1377

[21] Yoon, S., Kwak D., Implicit Navier-Stokes solver for three-dimensional compressible flows, AIAA Journal, Vol. 30, pages 2653-2659, 1992. C1387 